Keywords: Hanford, WTP, WRPS, Waste Transfer, DNFSB

Retention: Permanent

\title{
Test Plan - Solids Accumulation Scouting Studies
}

\author{
M.R. Duignan \\ T.J. Steeper \\ J.L. Steimke \\ M.D. Fowley
}

May 2012

Savannah River National Laboratory Savannah River Nuclear Solutions, LLC Aiken, SC 29808

Prepared for the U.S. Department of Energy under contract number DE-AC09-08SR22470. 
SRNL-STI-2012-00239

Revision 0

\section{DISCLAIMER}

This work was prepared under an agreement with and funded by the U.S. Government. Neither the U.S. Government or its employees, nor any of its contractors, subcontractors or their employees, makes any express or implied:

1. warranty or assumes any legal liability for the accuracy, completeness, or for the use or results of such use of any information, product, or process disclosed; or

2. representation that such use or results of such use would not infringe privately owned rights; or

3. endorsement or recommendation of any specifically identified commercial product, process, or service.

Any views and opinions of authors expressed in this work do not necessarily state or reflect those of the United States Government, or its contractors, or subcontractors.

\section{Printed in the United States of America \\ Prepared for \\ U.S. Department of Energy}


SRNL-STI-2012-00239

Revision 0

\section{REVIEWS AND APPROVALS}

AUTHORS:

M.R. Duignan, Engineering Development Laboratory

Date

T.J. Steeper, Engineering Development Laboratory

Date

J.S. Steimke, Engineering Development Laboratory

Date

M.D. Fowley, Engineering Development Laboratory

Date

TECHNICAL REVIEW:

D.J. Adamson, Engineering Development Laboratory

Date

T. A. Wooley, Waste Feed Technical Programs

Washington River Protection Solutions

Date

APPROVAL:

B.J. Giddings, Manager

Date

Engineering Development Laboratory

Savannah River National Laboratory

M.G. Thien, Manager - Waste Feed Technical Programs

Date

Washington River Protection Solutions 
SRNL-STI-2012-00239

Revision 0

\section{EXECUTIVE SUMMARY}

This plan documents the highlights of the Solids Accumulations Scouting Studies test; a project, from Washington River Protection Solutions (WRPS), that began on February 1, 2012. During the last 12 weeks considerable progress has been made to design and plan methods that will be used to estimate the concentration and distribution of heavy fissile solids in accumulated solids in the Hanford double-shell tank (DST) 241-AW-105 (AW-105), which is the primary goal of this task. This DST will be one of the several waste feed delivery staging tanks designated to feed the Pretreatment Facility (PTF) of the Waste Treatment and Immobilization Plant (WTP). Note that over the length of the waste feed delivery mission AW-105 is currently identified as having the most fill empty cycles of any DST feed tanks, which is the reason for modeling this particular tank.

At SRNL an existing test facility, the Mixing Demonstration Tank, which will be modified for the present work, will use stainless steel particles in a simulant that represents Hanford waste to perform mock staging tanks transfers that will allow solids to accumulate in the tank heel. The concentration and location of the mock fissile particles will be measured in these scoping studies to produce information that will be used to better plan larger scaled tests. Included in these studies is a secondary goal of developing measurement methods to accomplish the primary goal. These methods will be evaluated for use in the larger scale experiments.

Included in this plan are the several pretest activities that will validate the measurement techniques that are currently in various phases of construction. Aspects of each technique, e.g., particle separations, volume determinations, topographical mapping, and core sampling, have been tested in bench-top trials, as discussed herein, but the actual equipment to be employed during the full test will need evaluation after fabrication and integration into the test facility. 


\section{TABLE OF CONTENTS}

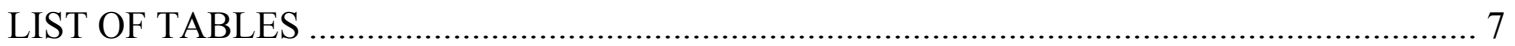

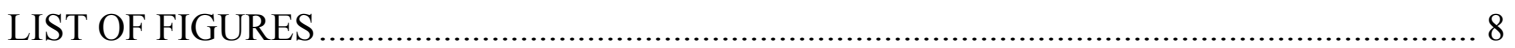



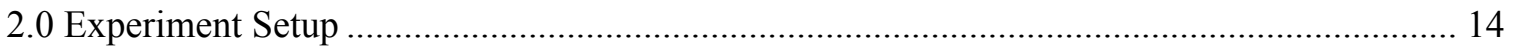

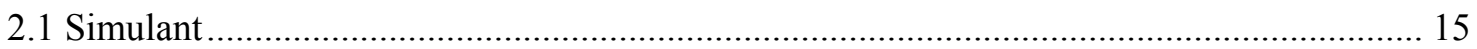

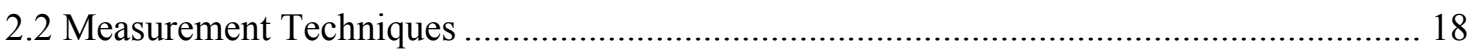

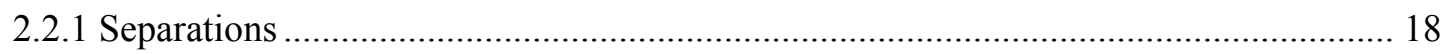



2.2.1.2 Separation of Gibbsite, Sand, and Zirconium Oxide................................................ 22



2.2.2.1 Vertical and Horizontal Profiles of Accumulated Solids .......................................... 25



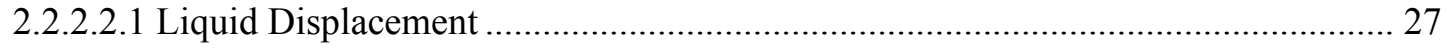

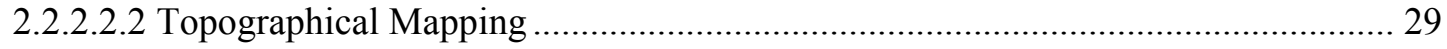

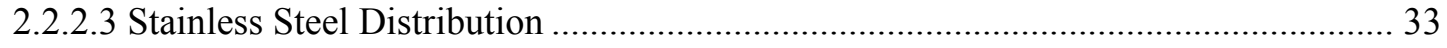

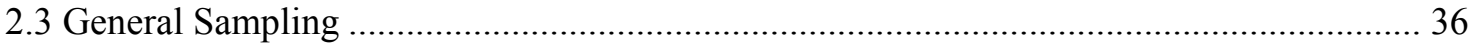

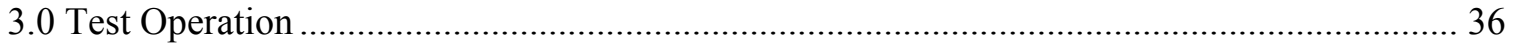



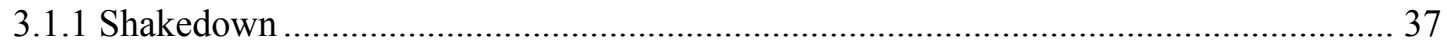



3.1.2.1 Determine removal efficiency of magnetic separator ............................................. 37

3.1.2.2 Show the overall operation with receipt tanks, magnetic separator, and solids

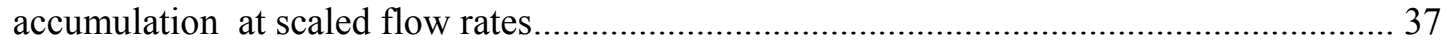

3.1.2.3 Feed rate to the MDT that will not disturb accumulated solids ................................. 38

3.1.2.4 Wait time between each FB to determine effect on accumulated solids .................... 39

3.1.2.5 Determine functionality and accuracy of laser height measurement........................... 39

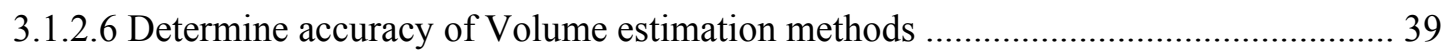

3.1.2.7 Determine functionality and of core sampler and its ability to capture a complete core

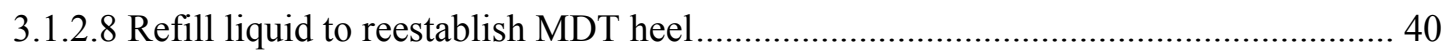

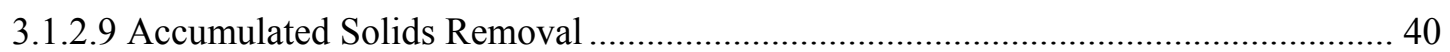

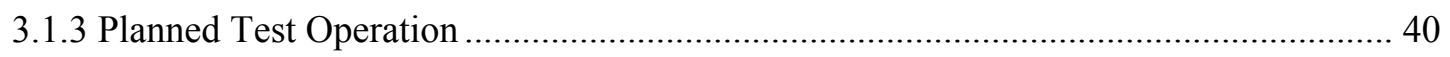

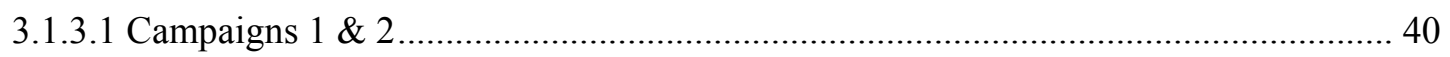




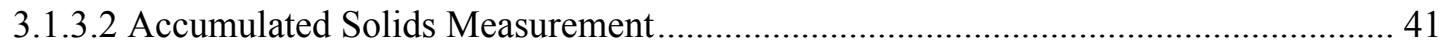

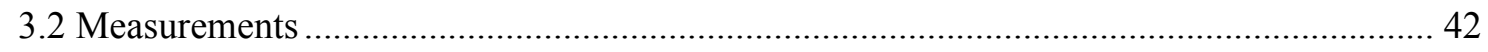

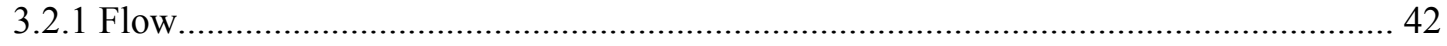

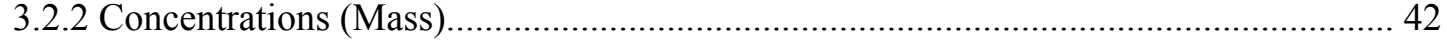

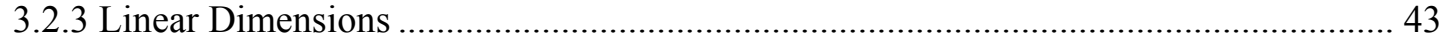



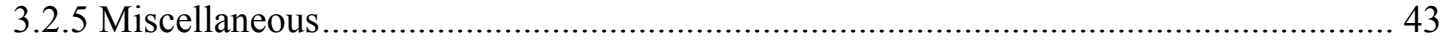

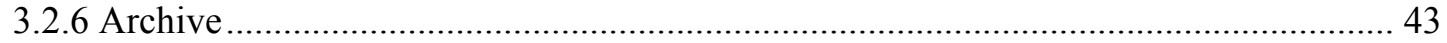





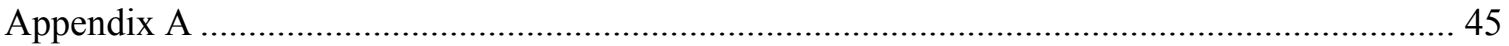




\section{LIST OF TABLES}

Table 1. Solids Accumulation Scouting Studies Test Objectives [3] ........................................ 14

Table 2. Concentrations of the supernatant used to suspended undissolved solids particles ....... 15

Table 3. Typical Conceptual Simulant as Modified for SRNL Testing ..................................... 16

Table 4. Comparison of full scale tank dimensions to those of the MDT ..................................... 36 


\section{LIST OF FIGURES}

Figure 1. The original SRNL 1/22-scale Mixing Demonstration Tank with its six batch receipt

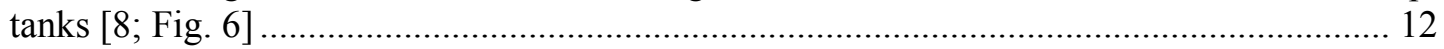

Figure 2. The modified flow schematic of overall solids accumulation test facility ................... 12

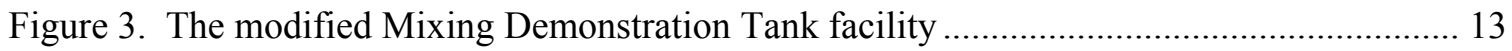

Figure 4. Properties of supernatant to be used for SRNL testing ............................................. 16

Figure 5. Particle sizes of SRNL simulant with four chosen UDS compared to the recommended [5] simulant 17

Figure 6. Settling characteristics of the SRNL simulant with four chosen UDS compared to the

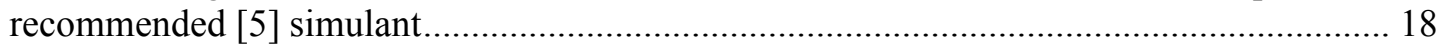

Figure 7. Bench-top test demonstrating the ability of separating stainless steel particles from water.

Figure 8. Magnetic Separations Device to Remove Stainless Steel Particles ............................. 21

Figure 9. 2.5-L bench-top settling test with full simulant after four days of settling .................. 23

Figure 10. Modified receipt tanks and compared those in Fig. 1 ............................................. 24

Figure 11. Typical Solids Heel in 1/22 Scale SRNL Mixing Demonstration Tank .................... 25



Figure 13. Positioner to location points of interest on the accumulated solids .......................... 27

Figure 14. Determining volume by measure volume of liquid displaced.................................. 28

Figure 15. Stereo camera system, using 1 color and 2 monochrome cameras (Note, that this photograph only shows an example of a camera system. What actually will be used is still being tested and it may be different.).

Figure 16. MDT with mock accumulated solids mounds. (Note the new drain in bottom center of the tank to lower the liquid level.) 30

Figure 17. Yellow sand mound in water to determine contrast between liquid and solids, which is

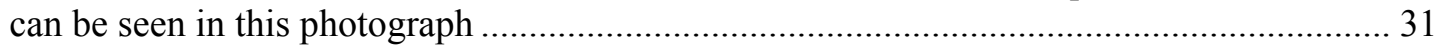

Figure 18. Contour plot of the solids mound shown in the preceding figure .............................. 31

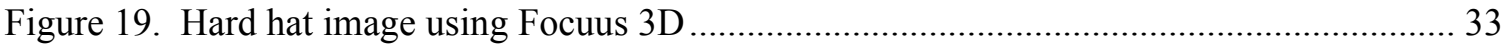

Figure 20. Mound of damp solids: ZrO2, Sand, and Stainless Steel Particles ............................ 34

Figure 21. Core sample drawn from solids shown in the preceding figure ................................ 34

Figure 22. Solids Sampler to Extract a Core of Accumulated Solids......................................... 35 


\section{NOMENCLATURE}

Campaign Completion of 10 complete cycles done at a single jet pump flow rate

Cycle One complete fill and empty of the MDT

DNFSB Defense Nuclear Facilities Safety Board

DST Double-Shell Tank

EDL Engineering Development Laboratory (Performing Organization)

ERPS E\&CPT Research Programs Section [E\&CPT=Environmental \& Chemical Process Technology]

FB Feed Batch (to WTP) - Approximately 145,000 gallons - 5 to 6.5 FB constitute one transferrable Hanford feed tank volume

FT Feed Tank - Where simulant will be prepared and fed to the MDT

JHA Job Hazards Analysis

Heel In the MDT the heel refers to the simulant at the bottom of the tank that is not transferred after each fill and empty cycle is complete. For SASS, it is estimated to be 3.23 inches in height and contains both liquid and accumulated solids. This scaled height is equivalent to the minimum DST mixer pump operating level of 72 inches.

HAP Hazards Assessment Package

HLW High (Radioactive) Level Waste

LSIT Large Scale Integrated Testing Program

MDT Mixing Demonstration Tank

PTF Pretreatment Facility of WTP

RT Receipt Tank - There are 7 receipt tanks, 6 will receive one Feed Batch (13.1 gal) and 1 will receive a half-Feed Batch (6.9 gal)

SASS Solids Accumulation Scouting Studies (This task)

SRNL Savannah River National Laboratory

SSSMD Small Scale Mixing Demonstration

WFD Waste Feed Delivery

WTP Waste Treatment \& Immobilization Plant 


\subsection{Introduction}

The objective of Solids Accumulation activities is to perform scaled testing to understand the behavior of remaining solids in a Double Shell Tank (DST) at Hanford during multiple fill, mix, and transfer operations that are typical of the High Level Waste (HLW) feed delivery mission [1]. As listed the Task Technical and Quality Assurance Plan [2], testing will focus on accumulation of total solids over time and the propensity for simulated fissile material to concentrate over time.

The overall Test Plan [3] of the Mixing and Sampling Demonstration Project [4] includes two scaled test platforms to evaluate the baseline design for mixing and transferring slurry from DST 241-AY-102, the first staged HLW feed, to the Waste Treatment and Immobilization Plant (WTP). However, AW-105, the second staged HLW tank, is being modeled for this test scope because current waste feed delivery planning shows it being utilized more than any other waste feed tanks over the life of the waste-treatment mission. The small scale test will be done at SRNL that constructed a 1:22-scale Mixing Demonstration Tank (MDT) to perform mixing and transfer studies. The objective of the SRNL test, referred to as the Solids Accumulation Scouting Studies (SASS) is to perform a series of Waste Feed Delivery (WFD) to WTP transfer and refill operations using the 1:22-scale MDT and evaluate the bulk material that remains in the tank. Testing will determine the amount of bulk solids remaining and the concentration and distribution of the fastest settling particles that accumulate in the tank heel ${ }^{1}$. Providing insight into how fast settling particles are distributed in a WFD feed staging tank is essential to criticality evaluations that include the accumulation of dense plutonium and uranium containing solids. The scope of the work is limited to preliminary scoping studies, the results of which will be used to define larger scale test work; to be performed using the test platform at Hanford.

Concurrent with this activity is the selection of appropriately complex simulants that are integrated with WTP simulant selection and supported by accurate analytical techniques to characterize the material of interest. This includes using simulants characteristic of the Hanford tank waste and following the recommended guidelines in Lee et al. [5].

However, the exact simulant to be used for SASS is listed in a simulant development plan [6] that was agreed to by the overall test program management. The test will also include sampling techniques [6] for characterizing the residual tank waste solids that accumulate in the tank after a series of transfer and refill operations.

The operation of SASS described in this test plan will use the MDT platform shown in Fig. 1 to perform a DST transfer campaign to characterize the solids that remain in the tank after a series of tank transfers have been performed. A DST transfer campaign includes a series of fill and transfer operations with the MDT. A transfer operation is completed when six and one-half batches of slurry are transferred from the MDT to the seven receipt tanks. The number of batch transfers is primarily based on full-scale operation. The volume of each batch is based on Interface Control Document-19 which requires full scale batches of 145,000 gallons to be sent to WTP. Since AW-105 has approximately 946,000 gallons of transferrable waste then the total number of batches is

\footnotetext{
${ }^{1}$ A heel is describe in the Nomenclature Section
} 
approximately 6.5 . The full tank volume of AW-105, i.e., $1,144,000$ gallons, is not transferrable because a minimum volume, referred to as a heel, is needed to maximize mixer pump operations. Following a successful transfer operation, the solids remaining in the heel of the MDT will be characterized and additional simulant will be added to refill the mixing tank. A series of transfer and refill operations, up to ten, will be performed in a campaign. The solids remaining in the tank after each transfer campaign will be characterized and compared to the total solids that are added during testing.

Initially SASS studies will identify a suitable complex simulant that is characteristic of Hanford tank waste. The complex simulant will contain slow settling, fast settling, and very fast settling solid particles and a simulated supernatant liquid phase. A method to characterize the quantity of very fast settling solids that are and are not transferred will be established so that monitoring the accumulation of very fast settling particles can be performed as successive transfer operations are completed. In selecting the very fast settling particles, particle size and density are expected to be the most important solids properties of the simulant. Particle shape is assumed to be less important but this will be confirmed during parallel testing studies by SRNL being done to support the WTP LSIT program and will be re-addressed, if necessary.

The 1:22-scale testing will be performed using the MDT test platform constructed at the SRNL testing facility. The MDT test platform was constructed to perform mixing and transfer demonstrations and has been used previously for WFD Mixing and Sampling Program testing work [7-9]. The MDT test platform will be used with modifications to perform the SASS studies to determine how fast settling particles are distributed in the tank heel after each transfer operation. Preliminary bench-top tests were and are being performed to develop sampling and analysis methods to characterize the heel. A schematic of the test facility the current exists before applying the modifications for the SASS is shown in Fig.1, a schematic of the overall flow system is shown in Fig. 2, and the modified piping and instrument drawing is Fig. 3. 


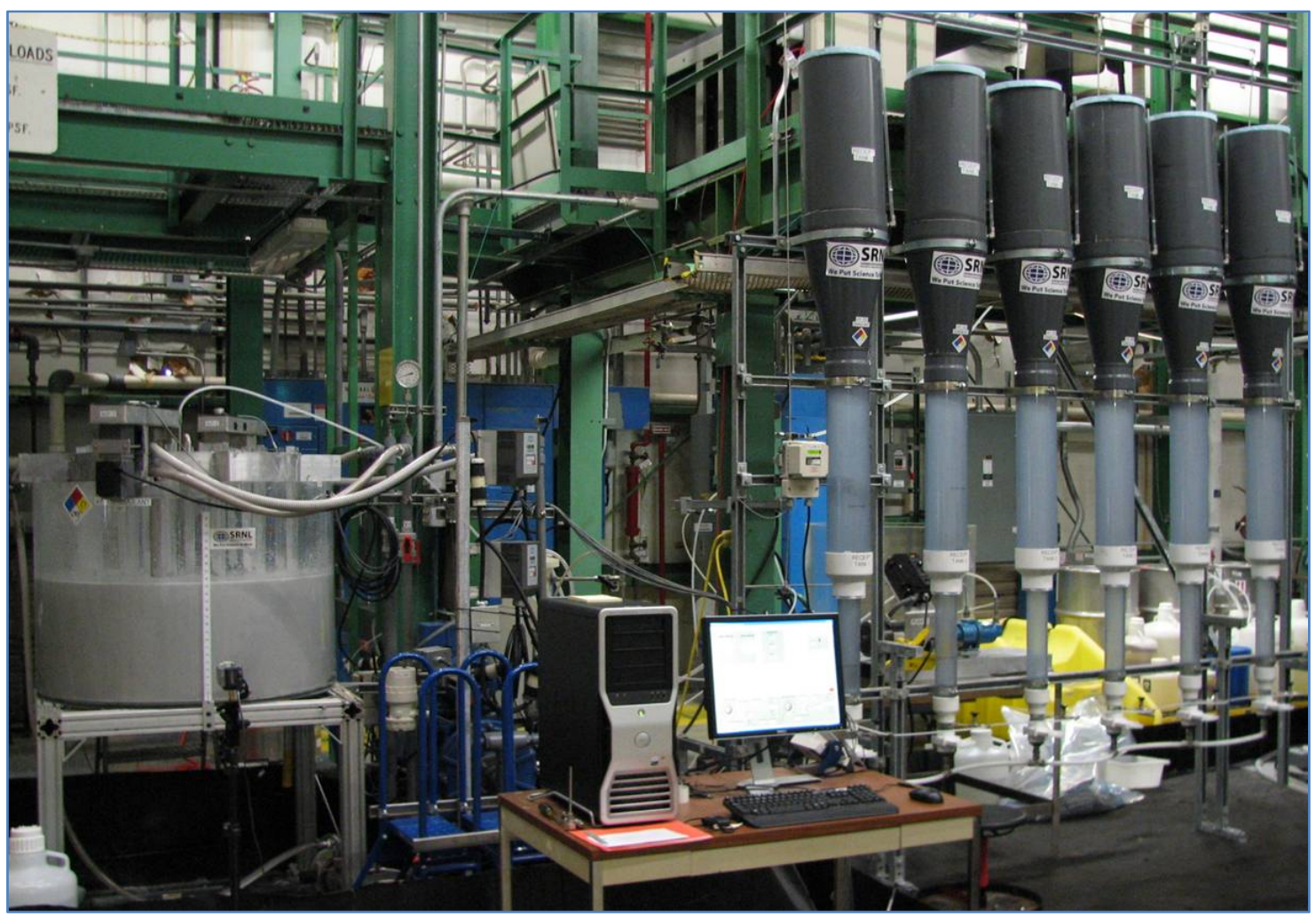

Figure 1. The original SRNL 1/22-scale Mixing Demonstration Tank with its six batch receipt tanks [8; Fig. 6]



Figure 2. The modified flow schematic of overall solids accumulation test facility 
SRNL-STI-2012-00239

Revision 0

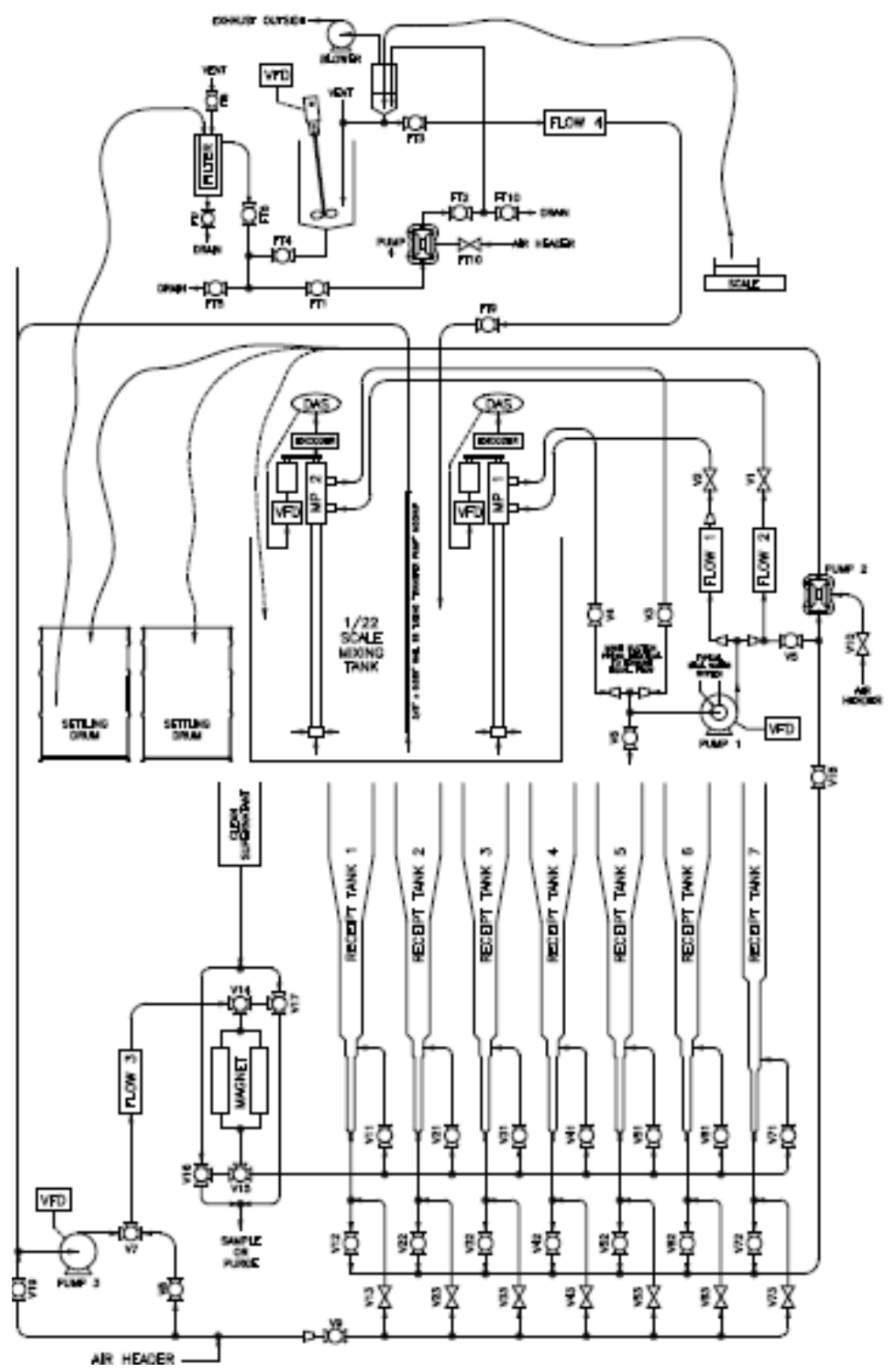

Figure 3. The modified Mixing Demonstration Tank facility 
The test objectives are summarized in Table 1 .

Table 1. Solids Accumulation Scouting Studies Test Objectives [3]

\begin{tabular}{|l|l|}
\hline Objective & Success Criteria \\
\hline $\begin{array}{l}\text { Demonstrate at two jet } \\
\text { nozzle velocities the } \\
\text { potential accumulation } \\
\text { of solids in the DST } \\
\text { after several transfer } \\
\text { and re-fill operations } \\
\text { are conducted. }\end{array}$ & $\begin{array}{l}\text { Mixing and transfer demonstrations are performed at two different } \\
\text { jet nozzle velocities with a base simulant that contains moderately } \\
\text { sized (approximately 100 microns), dense particles to represent } \\
\text { fissile material in the Hanford tank waste. The spike particles are } \\
\text { be exploited for quantification. }\end{array}$ \\
$\begin{array}{l}\text { The dense } 100 \text { micron particles that represent the very fast settling } \\
\text { particles that can accumulate inside a DST used for several staged } \\
\text { feeds are quantified relative to the amount of the solids added to the } \\
\text { tank. } \\
\text { The relative quantities of undissolved solids in each transfer batch } \\
\text { are estimated. } \\
\text { The accumulation of heel solids is evaluated after each tank volume } \\
\text { transfer. Visual changes will be noted and photographs will be } \\
\text { taken. } \\
\text { The accumulation of heel solids is quantified after the } 1^{\text {st }}, 5^{\text {th }} \text { and } \\
\text { characterize the } \\
\text { situal tank waste in- }\end{array}$ & $\begin{array}{l}10^{\text {th }} \text { tank volume transfer by measuring the volume of heel in the } \\
\text { tank. In the case where } 10 \text { transfer volumes are not performed } \\
\text { accumulation of heels solids is quantified at the } 1^{\text {st }}, 5^{\text {th }}, \text { and last } \\
\text { cycles. } \\
\text { Correlations relating the fraction of the mock very fast settling solids } \\
\text { transferred and remaining in the tank are evaluated with respect to } \\
\text { each transfer batch and after multiple tank volume transfers. }\end{array}$ \\
\hline $\begin{array}{l}\text { Techniques to sample and quantify the volume of residual solids are } \\
\text { identified and documented. } \\
\text { The error in the heel volume measurement is quantified. }\end{array}$ \\
\hline
\end{tabular}

\subsection{Experiment Setup}

This section will first discuss the simulated Tank waste, then the instruments to be used to take samples and evaluate the solids, and finally the current plan of operation of the test. 


\subsection{Simulant}

Along with the scaled test facility, the simulated waste is a principal part of the experiment. Details of the simulant is discussed the Simulant Development and Sampling Plan [6] but highlights and modifications are listed in his section.

In developing the simulant the starting point was to work with simulant material available from past SRNL testing [7-8] that included both a caustic supernatant and solids particles that would remain as undissolved solids (UDS) in the simulant, i.e., gibbsite and stainless steel particles. Based on published recommendations [5], and input from WRPS, the SASS project will use stored supernatant that has a density of $1.28 \mathrm{~g} / \mathrm{mL}$ and viscosity of $3 \mathrm{cP}$ and four solid particles of Gibbsite, Zirconium Oxide, Sand, and Stainless Steel Particles. The last particle is to represent the large density plutonium particles in waste and what will be measured in the accumulated solids to determine its deposited distribution. Furthermore, the solids loading range of the wastes to be fed to tank AW-105 is estimated at 0.44 to $203 \mathrm{~g} / \mathrm{l}$, with an average of $89 \mathrm{~g} / \mathrm{l}$. WRPS recommends using $100 \mathrm{~g} / \mathrm{l}$ for SASS and that is planned.

The supernatant is basically a Newtonian caustic solution with $\mathrm{pH}>14$. It contains several compounds to represent Hanford waste. Table 2 shows the approximate concentrations of those compounds.

Table 2. Concentrations of the supernatant used to suspended undissolved solids particles

\begin{tabular}{|c|c|c|}
\hline \multicolumn{3}{|c|}{ Chemical Composition for Supernatant } \\
\hline Compound Formula & Name & $\mathrm{g} /$ liter \\
\hline $\mathrm{NaAlO} 2 * \mathrm{H} 2 \mathrm{O}$ & Sodium Aluminate & 29 \\
\hline $\mathrm{NaOH}$ & Sodium Hydroxide & 24 \\
\hline $\mathrm{Na} 2 \mathrm{CO} 3$ & Sodium Carbonate & 66 \\
\hline $\mathrm{Na} 2 \mathrm{C} 2 \mathrm{O} 4$ & Sodium Oxalate & 0.8 \\
\hline KNO3 & Potassium Nitrate & 1.8 \\
\hline NaNO3 & Sodium Nitrate & 280 \\
\hline $\mathrm{NaNO} 2$ & Sodium Nitrite & 36 \\
\hline $\mathrm{Na}_{2} \mathrm{SO} 4$ & Sodium sulfate & 18 \\
\hline $\mathrm{Na} 3 \mathrm{PO} 4 * 12 \mathrm{H} 2 \mathrm{O} * 1 / 4 \mathrm{NaOH}$ & Trisodium Phosphate & 18 \\
\hline $\mathrm{NaCl}$ & Sodium Chloride & 4 \\
\hline $\mathrm{NaF}$ & Sodium Fluoride & 0.4 \\
\hline
\end{tabular}

This liquid simulant is excess simulant from a previous test [10], but was also used for past MDT tests [7-8] and was deemed acceptable the WRPS to use for the present test. Because there are at least eight 300 gallon totes of supernatant in storage at SRNL it is immediately available at no charge. Its chemical composition has been developed to represent typical for Hanford waste tank supernatants [10]. As shown in Fig. 4, the totes contain a range of liquid densities from 1.25 $\mathrm{g} / \mathrm{mL}$ to $1.37 \mathrm{~g} / \mathrm{mL}$ and a range of viscosities from 2.5 to $6.5 \mathrm{cP}$. Note that SRNL will blend 
material from totes \#3 and \#4 to obtain sufficient supernatant with properties similar to the supernatant previously tested, density $\sim 1.29 \mathrm{~g} / \mathrm{mL}$ and dynamic viscosity $\sim 3.3 \mathrm{cP}[7-8]^{2}$.



Figure 4. Properties of supernatant to be used for SRNL testing

As for the solids, it was decided between WRPS and SRNL on 2/29/2012 that SASS simulant will have a UDS composition approximately to what is shown in Table 3.

Table 3. Typical Conceptual Simulant as Modified for SRNL Testing

\begin{tabular}{|c|c|c|c|}
\hline SASS Component & $\begin{array}{c}\text { Density } \\
(\mathrm{g} / \mathrm{ml})\end{array}$ & $\begin{array}{c}\text { Median particle size } \\
\text { by volume, } \mu \mathrm{m}\end{array}$ & $\begin{array}{c}\text { Mass percentage in } \\
\text { undissolved solids (2) }\end{array}$ \\
\hline gibbsite & 2.42 & 11 & 71 \\
\hline Safety yellow sand & $2.4(1)$ & 293 & 13 \\
\hline zirconium oxide & 5.7 & 15 & 15 \\
\hline stainless steel & 8 & 125 & 100 \\
\hline Total Mass \% & & & 100 \\
\hline
\end{tabular}

(1) The sand itself has a density closer to $2.65 \mathrm{~g} / \mathrm{mL}$, but it is coated with a colored resin that is lighter and giving the small density of approximately $2.4 \mathrm{~g} / \mathrm{mL}$.

(2) The UDS loading will be $100 \mathrm{~g} / \mathrm{l}$.

\footnotetext{
${ }^{2}$ In Table 1 of Refs. 7 and 8 the viscosity value is given as $2.55 \mathrm{cP}$, but the units were in error and they should have been 2.55 centistokes, a kinematic viscosity; therefore, the actual dynamic viscosity was $3.3 \mathrm{cP}$.
} 
The particle size distributions (PSD) of the UDS can be found in App. A. These particles show an overall PSD similar to that of the Hanford Typical Conceptional Simulant [5], Fig. 5.

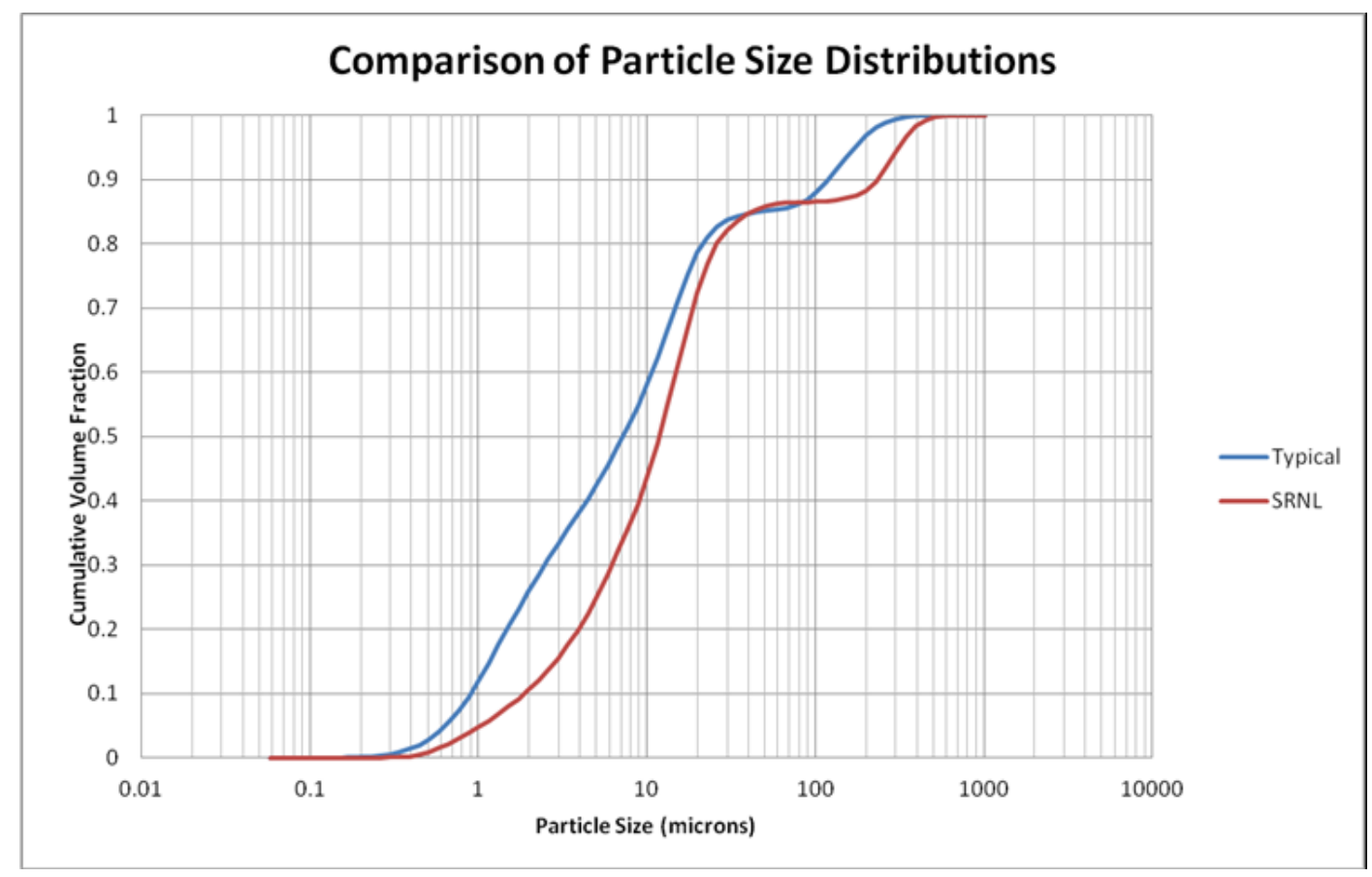

Figure 5. Particle sizes of SRNL simulant with four chosen UDS compared to the recommended [5] simulant

When comparing the distribution of Archimedes number (Ar) of the SASS simulant to the Typical Conceptual Simulant [5] they are equivalent, Fig. 6. Note that the Ar is a dimensionless number, Eq. (1), that is useful to compare the settling rates of different suspension slurries, i.e.,

$$
\operatorname{Ar}=\frac{\left(\frac{\rho_{\mathrm{S}}}{\rho_{\mathrm{L}}}-1\right) \mathrm{gd}^{3}}{v^{2}}
$$

where $d$ is the particle diameter, $\rho_{\mathrm{S}}$ is the UDS density, $\rho_{\mathrm{L}}$ is the liquid density, $v$ is the kinematic viscosity of the liquid, which is the dynamic viscosity divided by the density of the liquid, and $\mathrm{g}$ is the gravitational constant. 




Figure 6. Settling characteristics of the SRNL simulant with four chosen UDS compared to the recommended [5] simulant

\subsection{Measurement Techniques}

In performing mock transfers of feed batches (FB) the simulated waste will be analyzed to determine the concentration and distribution of stainless steel (SS) particles in accumulated solids on the bottom of a 1/22-scaled Hanford Tank Farm staging tank AW-105, the MDT. To make measurement several techniques were develop for this task. They are explained below to understand the method of operation.

\subsubsection{Separations}

One method to determine what is left in the staging tank after transferring waste is by subtracting the amount of material transferred from the tank from the amount of material initially in the tank. In this experiment the simulated staging tank will be filled with known amounts of supernatant and undissolved solids (UDS), including stainless steel particles that represent plutonium particle in the real waste. Measuring stainless steel solids as they leave the tank will allow the remaining stainless steel solids to be estimated. Of secondary important is measuring the quantity of the other solids that are transferred and they will be estimated as described in the following sections.

\subsubsection{Separation of Stainless Steel Particles}


One method to determine how much stainless steel (SS) is left in the accumulated solids is knowing how much was transferred out of the tank. In fact, knowing the concentration of simulated plutonium particles in each transfer to WTP can be useful. Therefore, a method was developed to accomplish this separation and it entails the magnetic separation of slightly ferric stainless steel particle from all other solid particles in the test simulant.

As can be seen in the flow diagram, Fig. 2, the simulant will be transferred through a magnetic separator before continuing on to one of the receipt tanks. After the transfer of each FB the magnets will be removed from the separator to collect and measure the mass of the steel particles.

The SS powder that was used previously [7-8] during the transfer tests is fairly magnetic. A bench-top test has shown, Fig. 7, that the larger particles are drawn across an inch or more of water to the side of a plastic bottle within a couple seconds using a strong magnet (1" OD x $1 / 4$ " thick neodymium-iron-boron, $14 \mathrm{lb}$ pull). Fine particles take appreciably longer to be collected.
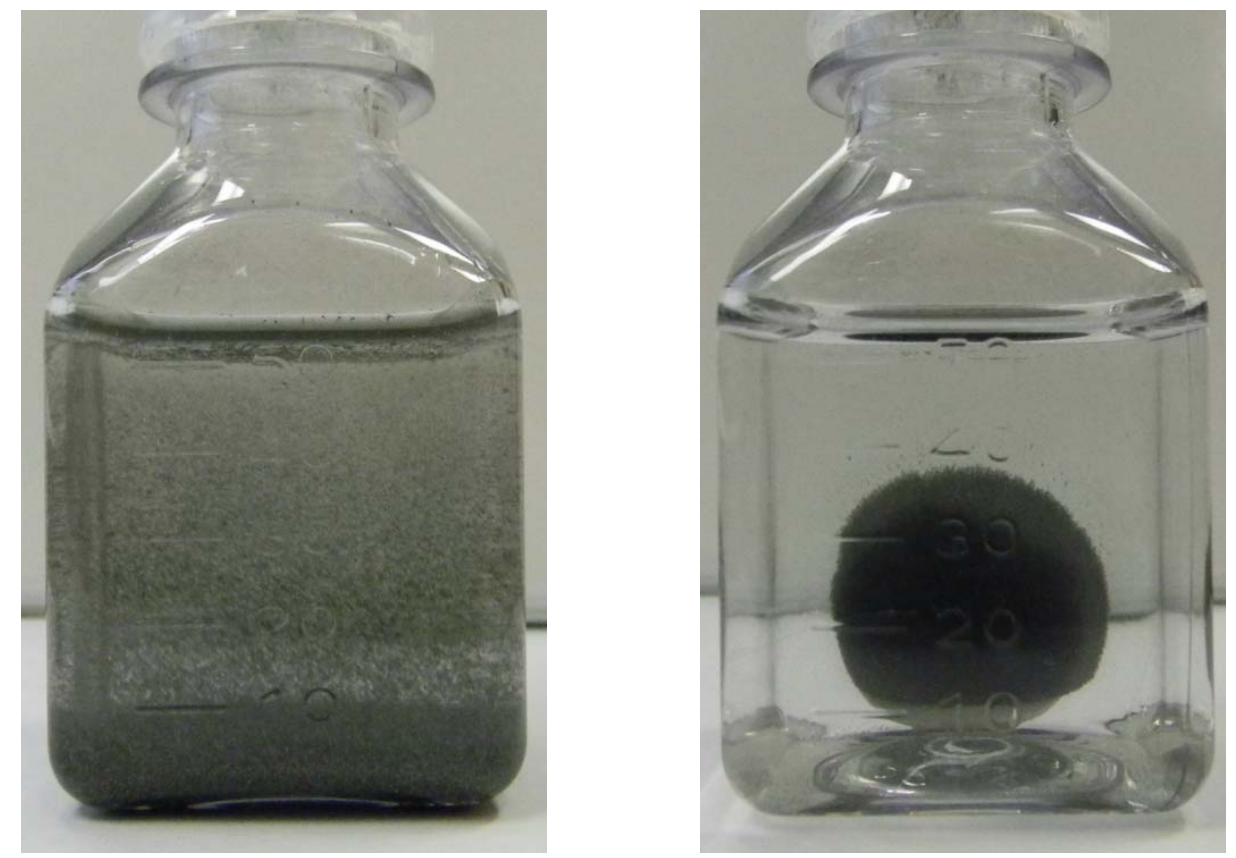

\section{Figure 7. Bench-top test demonstrating the ability of separating stainless steel particles from water}

The attractive force should be proportional to the mass of the particle which increases as the cube of the diameter. The flow resistance should be proportional to the area which increases as the square of the diameter. Sieving the SS to remove fines will improve the separation efficiency in any technique involving a limited magnet exposure time, such as separation from a flowing stream. The fines should not be important when using a magnet to separate the SS during long term exposure such as would be expected in individual sample separations. In any case, a few mass percent of fines can probably be ignored as the error will be small.

The concept for separating the SS from the other constituents of the simulant in small samples is very simple. A magnet will be placed on top of a thin plastic cover over a shallow clear sample container like a petri dish. The sample will be sealed in the container and gently shaken to 
suspend the solids. The SS will be collected beneath the lid adjacent to the magnet. After sufficient time, all of the SS should have been collected. The lid will be removed and placed over a pre-weighed filter paper, and the magnet will be removed allowing the SS to fall to the filter paper. After drying, the filter paper with SS will be weighed to determine the amount of SS in the sample. This method will be tested by processing a sample of prototypical simulant with a known amount of SS particles.

The concept for separating the SS from the simulant that is pumped out of the mixing tank during transfers is much more complicated since there is a limited amount of time to effect the separation. Based on our simple bench tests with water, most of the SS particles can be removed from a 1" flow channel in less than 3 seconds. If transfer continues through a 0.19 " by 4.75 " flow channel, at the historical rate of $0.58 \mathrm{gpm}$, the velocity will be $0.20 \mathrm{ft} / \mathrm{s}$. If the flow channel is adjacent to strong magnets for 12 " of its length, the time to pass the entire magnetic surface will be 5 seconds.

The packing density for very loose random packing of uniform size spheres as about $56 \%$ solid [11]. Tapping and vibration will increase the packing density up to a maximum of about $64 \%$ solid. The packing density for a mixture of sphere diameters will be higher. For estimating purposes, assume the packing density of SS particles on the surface of the flow channel adjacent to the magnets is $50 \%$. Splitting the flow channel into two segments with half on each side of the magnets will provide twice the surface area for collection of particles. For each fill cycle we will add about 85.7 gallons of simulant with 100 grams of UDS per liter, or (85.7 gal)(3.7854 $1 / \mathrm{gal})(100 \mathrm{~g} / \mathrm{l})=32,441 \mathrm{~g}$ of UDS. Using a concentration of $1 \%{ }^{3}$ by weight is SS, or $324 \mathrm{~g}$. Solid SS has a density of about $8 \mathrm{~g} / \mathrm{ml}$. Noting that there will be a total of 6.5 transfers per cycle, then with all these assumptions and known facts, the average thickness of the layer of collected SS powder on the flow channel walls adjacent to the magnets for each transfer is shown in Eq. (2):

$$
\mathrm{t}=\frac{(324 \mathrm{~g})(\mathrm{in} / 2.54 \mathrm{~cm})^{3}}{(6.5 \text { transfers })\left(8 \mathrm{~g} / \mathrm{cm}^{3}\right)(0.50 \text { packing density })(4.75 \mathrm{in})(12 \mathrm{in})}=0.013 \mathrm{in}
$$

This analysis provides the basis for the conceptual design of a magnetic separator for use during the transfers shown on the next page. It has two flow channels, see Fig. 8, one on each side of an aluminum plate containing six 2 in $^{2}$ by $1 / 4$-in thick. neodymium-iron-boron magnets ( $37 \mathrm{lb}$ pull).

\footnotetext{
${ }^{3}$ This amount is arbitrary but agreed to by WRPS. The stainless steel is to represent the heavy plutonium oxide particle in Hanford waste.
} 
SRNL-STI-2012-00239

Revision 0


Figure 8. Magnetic Separations Device to Remove Stainless Steel Particles 
The channels are $23 / 8$-in wide and taper from a 7/16-in depth at the top to about 3/16-in depth at the bottom. To prevent plugging, the flow enters the top of the separator so the particles will continue to fall downward even though the flow velocity is small. The flow channel is larger at the top to help even out the thickness of the layer of SS particles collected at the magnets. The "teeth" at the top of the flow channel are intended to help spread out the flow across the width of the flow channel. The "teeth" are pointed to avoid piling up solids that might cause plugging. The depth of the flow channel at the top is reduced to about $1 / 8$-in to provide a larger flow velocity above the magnet region to further reduce the chance of plugging. The inner surface of the flow channel will be a thin (0.015-0.020 in) plastic film sealed around the edge with an O-ring to a central aluminum frame. The cover plates will be acrylic to allow visual observation of the separation. The magnets are glued into an aluminum plate that can be removed from the device by sliding the plate upward, thus releasing the SS particles for draining into a sample container.

This separator will be fabricated and tested by pumping through a prototypical mixture of simulant solids and supernatant at the desired transfer rate (assumed to be $0.58 \mathrm{gpm}$ ). The amount of SS particles collected will be compared to the known amount added to the simulant to determine the capture efficiency. The goal, of course, is $100 \%$ capture efficiency.

\subsubsection{Separation of Gibbsite, Sand, and Zirconium Oxide}

Once the stainless steel particles are removed from a single FB of simulant and the batch is contained within one of the 7 receipt tanks, Fig. 2, the rest of the particles will be observed for settling heights. As seen from a bench-top settling test, Fig. 9, which used 2.5 liters of test simulant, at the very least the level of sand is readily detectable within a few minutes after material is added. In Fig. 9 the sand is blue, but in the actual test it will be a bright yellow.

A second bench-top test with just sand in supernatant, in the same diameter tube and with the same mass of sand, resulted in a very similar height of sand indicating that the small particles of other materials in the interstitial voids do not significantly affect the its settle volume. That is, the receipt tanks will be useful to estimate the mass of sand transfer in each batch but, unfortunately, to estimate the mass of Gibbsite because the average-size 11-micron particles took many days to completely settle. The zirconium oxide particles seemed to settle relatively fast too, but because of it very light coloring it is much harder to distinguish from the Gibbsite. However, a time will be chosen to wait after each complete transfer to roughly estimate the total batch of solids in each batch. It should be sufficient to determine if the volume of solids batch-to-batch changes and this bench-top test was used to redesign the reduced-volume receipt tanks, which are shown in Fig. 10. 
SRNL-STI-2012-00239

Revision 0

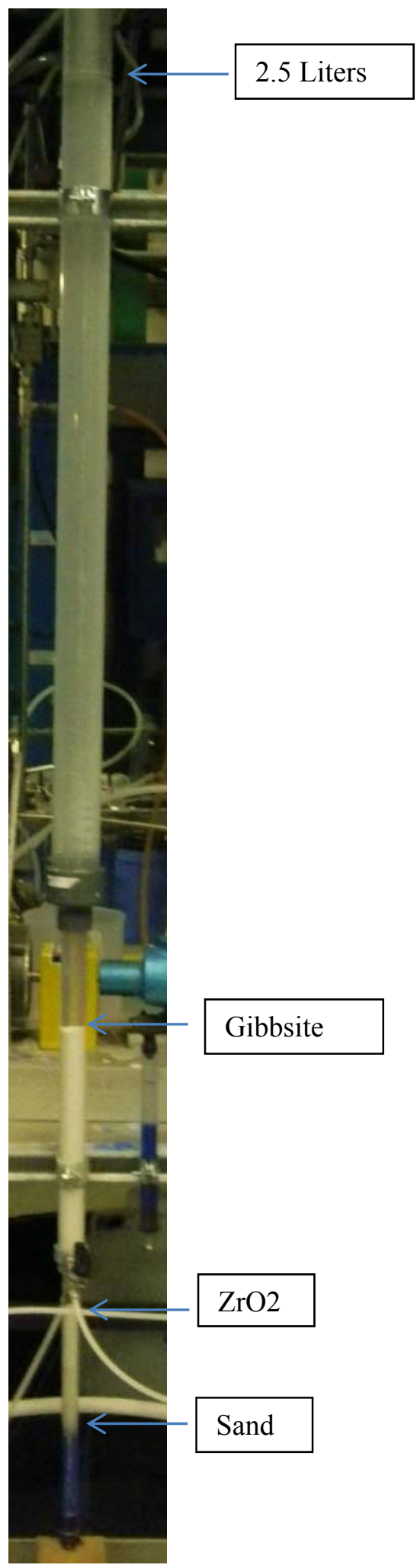

Figure 9. 2.5-L bench-top settling test with full simulant after four days of settling 
SRNL-STI-2012-00239

Revision 0

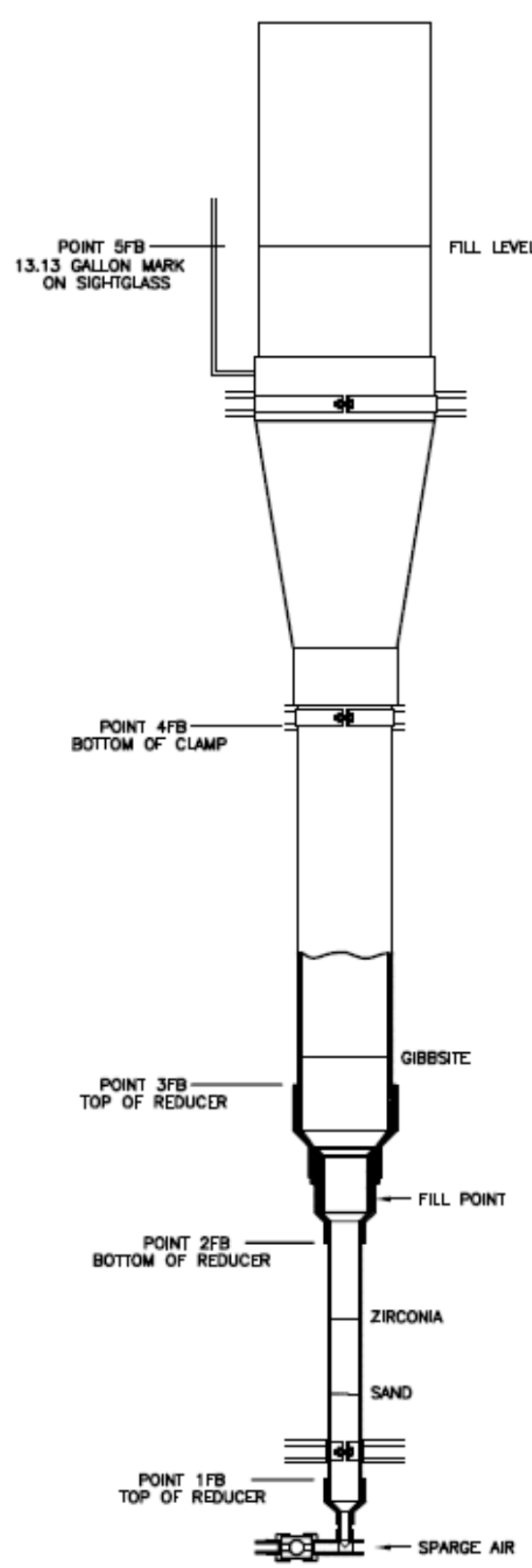

FULL BATCH

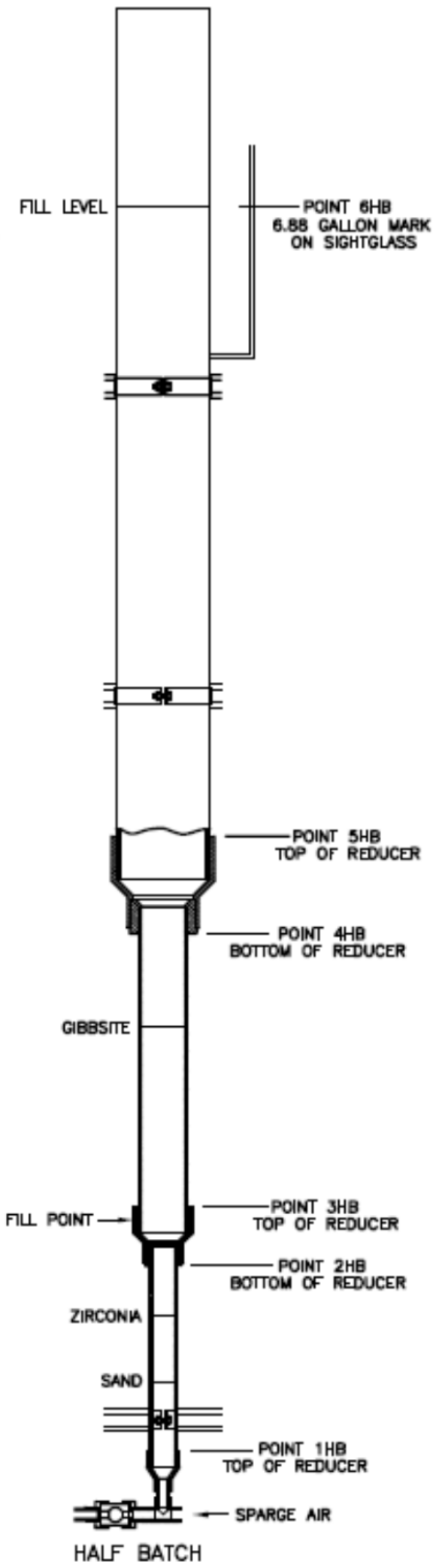

HALF BATCH

Figure 10. Modified receipt tanks and compared those in Fig. 1 


\subsubsection{In Tank Accumulated Solids Measurements}

Consideration was given to several techniques that can profile and measure the volumes of accumulated solids, e.g., Fig. 11. Note that the volume measurements will not quantify the speciation of the solids. The first three techniques listed below will be used (1. Measurement of the volume of discrete layer of accumulated by displacement of liquid, 2. Photographic data to obtain contour plots of the settled solids, and 3. Vertical height measurements of the mounds of settled solids to be used in conjunction of technique 2 to relate volumes to the contour maps and compare to the values obtained in technique 1). The fourth, and last technique, a laser scan of the entire bed of settle solids, to determine the volume profile of settled solids, will be only a onetime demonstration. Its prohibitive cost does not allow for its inclusion for the entire task, but it is included so that it can be evaluated for future incorporation as the need arises and funding become available.

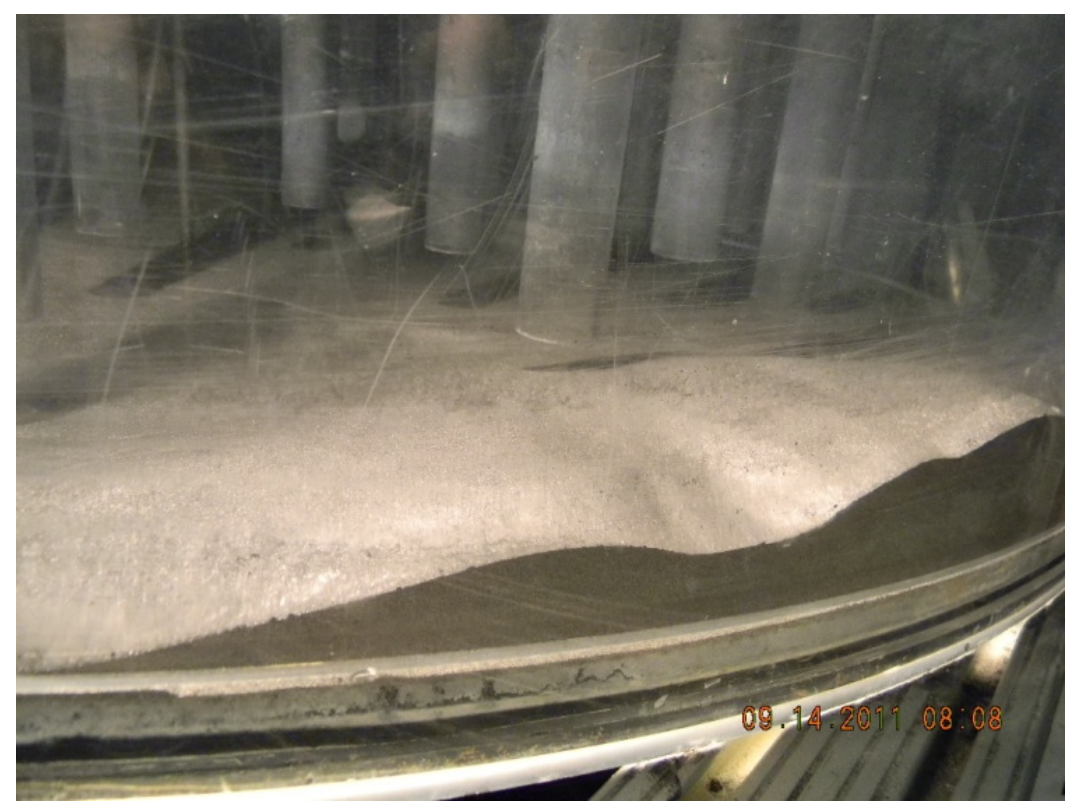

Figure 11. Typical Solids Heel in 1/22 Scale SRNL Mixing Demonstration Tank

\subsubsection{Vertical and Horizontal Profiles of Accumulated Solids}

When the MDT has transferred its 6.5 FBs what remains will be a 3.23-inch heel of liquid and solids that represents the full-scale heel of 72 inches. At that point measurements can take place to estimate the volume of the accumulated solids. The first ${ }^{4}$ step in this process is determining the shape of the settle solids with the use of distance laser probes.

\footnotetext{
${ }^{4}$ The distance measuring lasers can measure surfaces through clear liquids, but they may be challenged with the simulant supernatant if the unsettled solids, expected to be mostly gibbsite and some $\mathrm{ZrO}^{2}$, settle too slow and do not allow the lasers to function in a timely manner. If this is the case, this technique will only be used when the solids are exposed by lowering the liquid level for the other measurement techniques.
} 
Several laser distance meters will be used in conjunction with a positioning system to take measurements of the heel at numerous points along a grid. The measurements along the grid will establish the contour map of the solids heel. Figure 12 shows top view of a laser positioning system that is being fabricated to place laser distance meters at numerous points chosen to coincide with important features (high points, borders, leveled section, etc.) of the settled solids. Figure 13 show an isometric of the planned device.

The positioning system will be controlled by the PC controller to move and place the lasers in place. The lasers outputs will be sent to the test computer to automatically measure and record the distance at each grid point. The distance measurements will be used to define the contour of the solids heel and determine the volume of the solids heel.

The grid will not cover the entire surface of the tank bottom due to the two jet mixers in the tank. However, it is expected that the entire solids heel will be in the portion of the tank bottom within the grid.

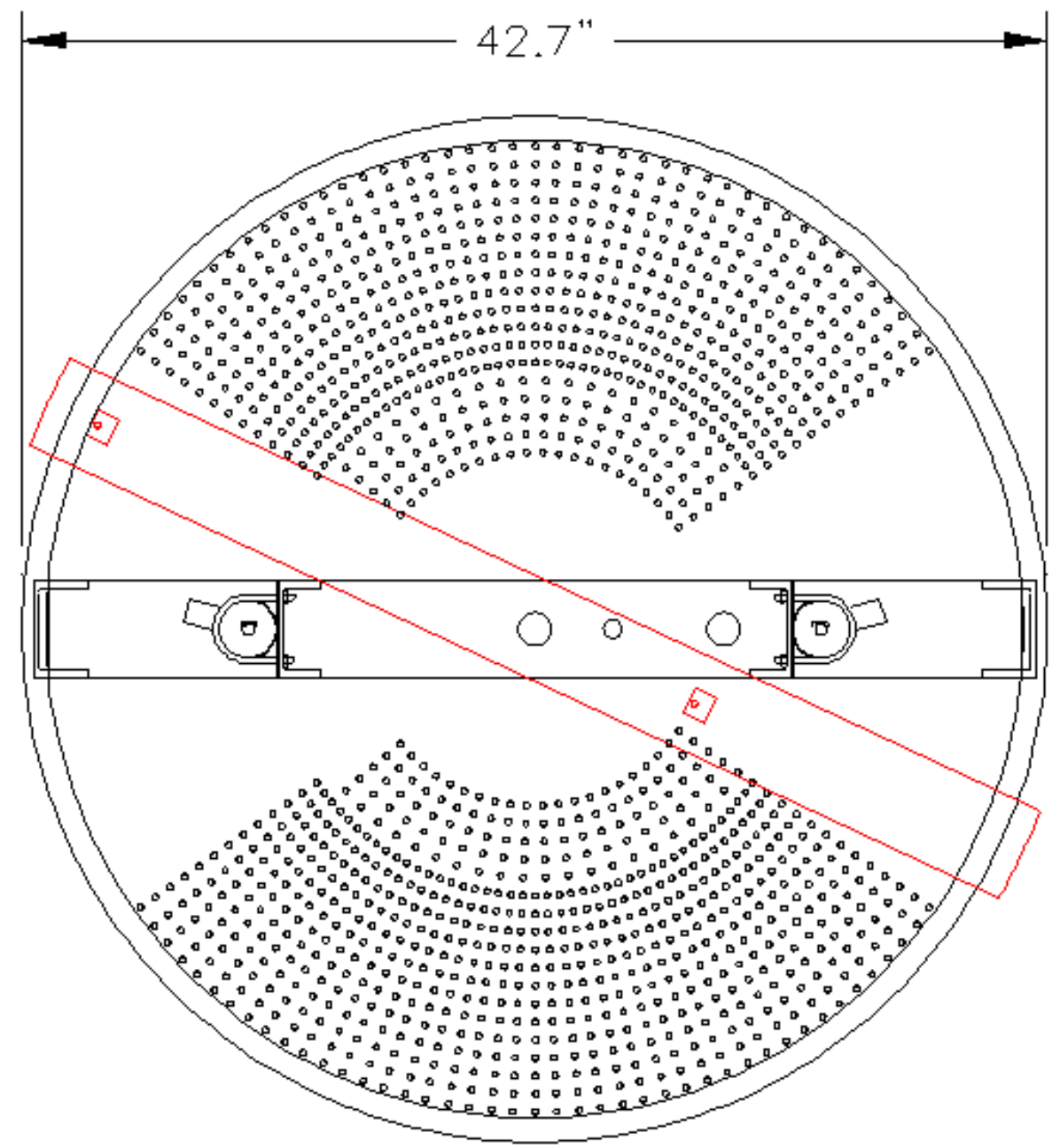

Figure 12. Proposed grid of measurement points 




Figure 13. Positioner to location points of interest on the accumulated solids

\subsubsection{Volume Determination}

The volume of the accumulated solids will be estimated by two methods: Liquid Displacement and Laser Scanning.

\subsection{Liquid Displacement}

When only the heel volume remains in the tank, and the test is at one of the accumulated solids measurement cycles currently planned as the $1^{\text {st }}, 5^{\text {th }}$, and last cycle for each of the two campaigns, then the volume estimation will begin.

The heel level will be drained to just above the settled solids and then be drained slowly in increments of liquid height, e.g., $1 / 8^{\text {th }}$ in., $1 / 4^{\text {th }}$ in., $3 / 16^{\text {th }}$ in., $1 / 2$ in., etc. to the bottom of the tank. 
The volumes will be estimate by the volume of displaced supernatant. That is, the tank volume, without solids, will be calibrated at those increments for baseline volume. To drain the tank to those increments a drain hole will be drilled into the bottom of the tank in a location where solids are not expected to accumulate. A short drain tube with a valve will be installed in the hole. The valve will closed and flush with the bottom of the tank while mixing, but during the heel measurements, with no mixing, the valve will be slowly raised and opened to minimize the introduction of suspended solids. Liquid will be drained from the tank into a graduated container for volume and weight measurements.

In bench-top tests this method was shown to be quite accurate. Figure 14 shows a plastic cone in a small container. The measured volumes of the cone segments were better than $1 \%$ to the known volumes, but for irregular shaped solids mounds the measurement uncertainty will be larger.

The increments can be established by several means and the most accurate method can be found from preliminary testing and uncertainty analyses:

- Tape measure affixed to the outside of the tank in one or two places. The level is read through tank wall.

- Graduated dipstick in the tank. The level read from graduations on dip stick.

- Conductivity probe on a telescoping, graduated dip stick. Level determined by lowering dip stick into tank until liquid is detected. Graduations at the top of the stick will be correlated to the depth of the liquid.

- Laser distance meter reading the position of a float in the liquid.
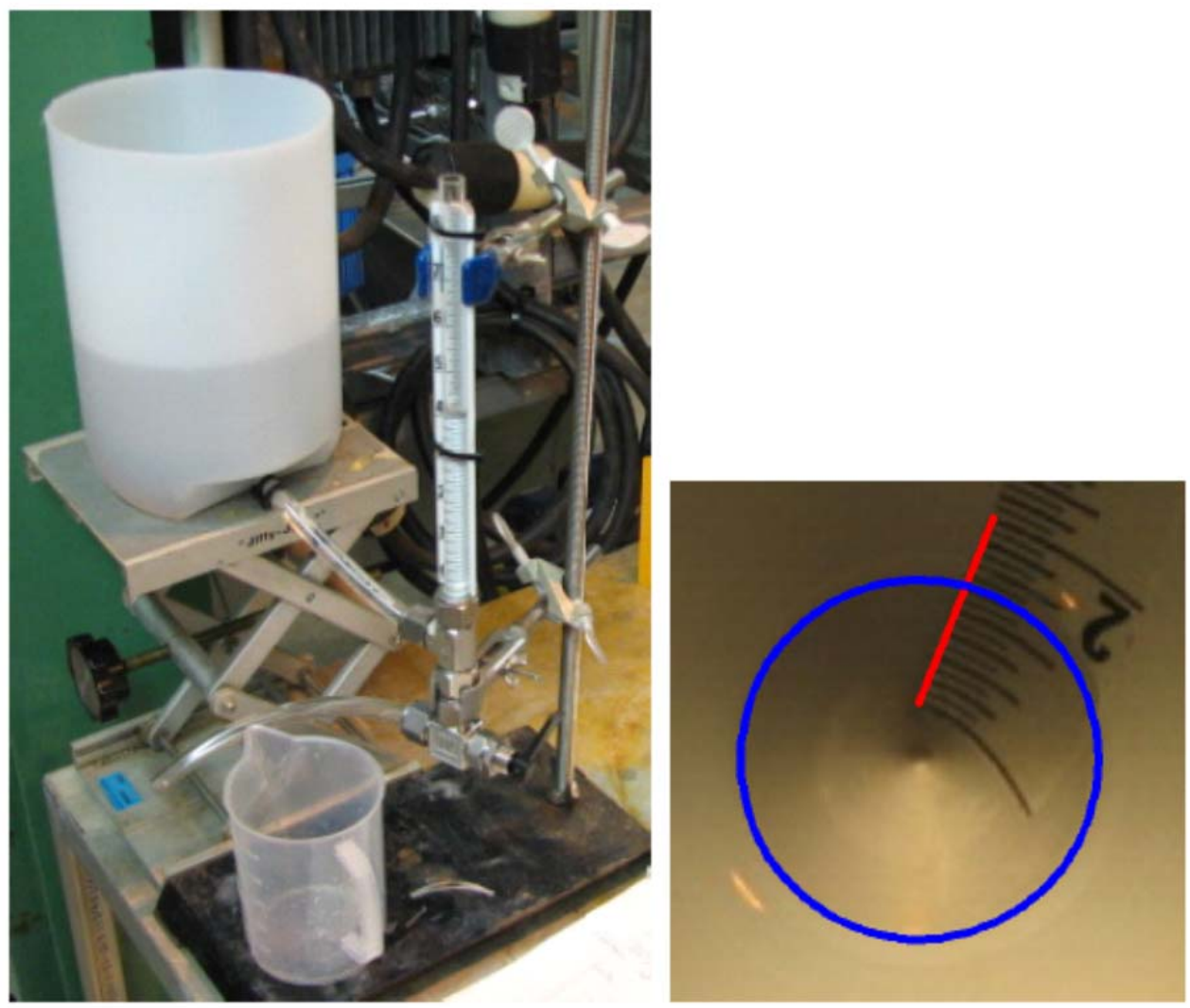

Figure 14. Determining volume by measure volume of liquid displaced 


\subsection{Topographical Mapping}

A simple visual-scanning method, referred to as a stereo vision system, already employed in the past at SRNL [11] will be utilized to map the accumulated in the MDT. Overhead photos of the exposed solids heel, taken during the draining process described above, can provide a contour map of the tank heel. The volume of the heel can be derived from the contour map. A camera system, an example is shown as Fig. 15, will be rigidly positioned above the tank and pictures of the exposed heel can be photographed as the tank is incrementally drained. This can be done in conjunction with the process described in the previous Section 2.2.2.2.1. The cameras will be placed at a height above the tank, to be determined during pretesting, e.g., Fig. 16, so that 3-D effects do not skew the estimation of exposed solids in the 2-D photograph.

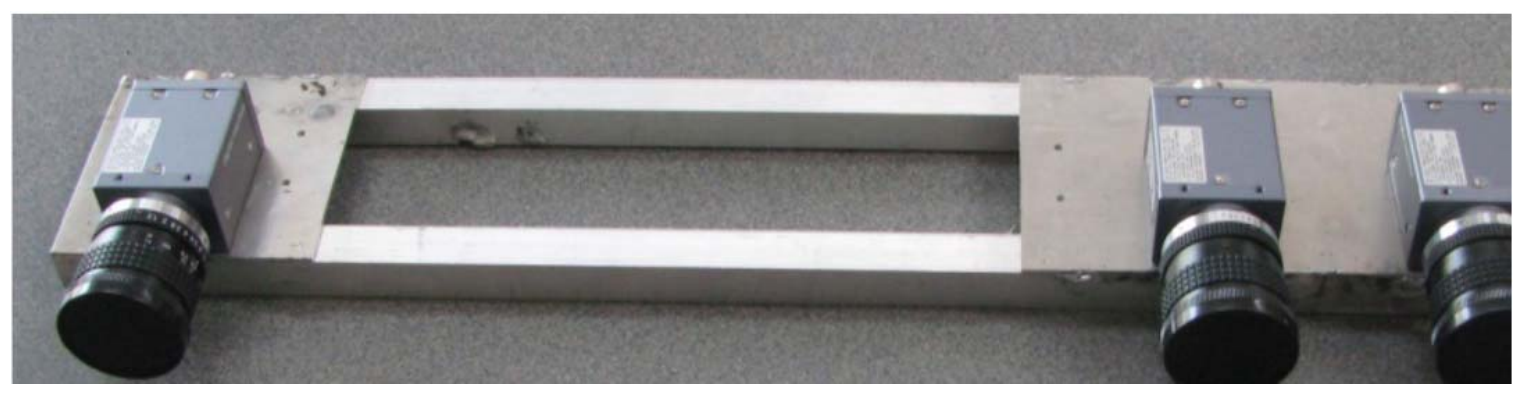

Figure 15. Stereo camera system, using 1 color and 2 monochrome cameras (Note, that this photograph only shows an example of a camera system. What actually will be used is still being tested and it may be different.)

As explained in Marzolf and Folsom [12], "A stereo vision system is a set of two or more cameras used to extract depth of a 3D scene as viewed from different vantage points and modeled after binocular vision in humans. Binocular vision is defined as vision from two eyes where the data being perceived from each is overlapped. The overlap from the two different views is used to perceive depth. Stereoscopic vision is the use of binocular vision to perceive a three dimensional structure. A stereo vision system is a set of two or more cameras used to extract depth of a 3D scene as the stereo system sends a left and right image of the 3D scene being viewed to a stereo vision system software program. The software rectifies the images, correlates the pixels, and re-projects the $2 \mathrm{D}$ points to a $3 \mathrm{D}$ point cloud. A point cloud is simply a set of $x, y$, $z$ coordinates extracted from the $x, y$ pixel coordinates and the disparity associated with each point. In addition, the color image taken by the stereo camera can be then be overlaid onto of the point cloud creating a texture map." 


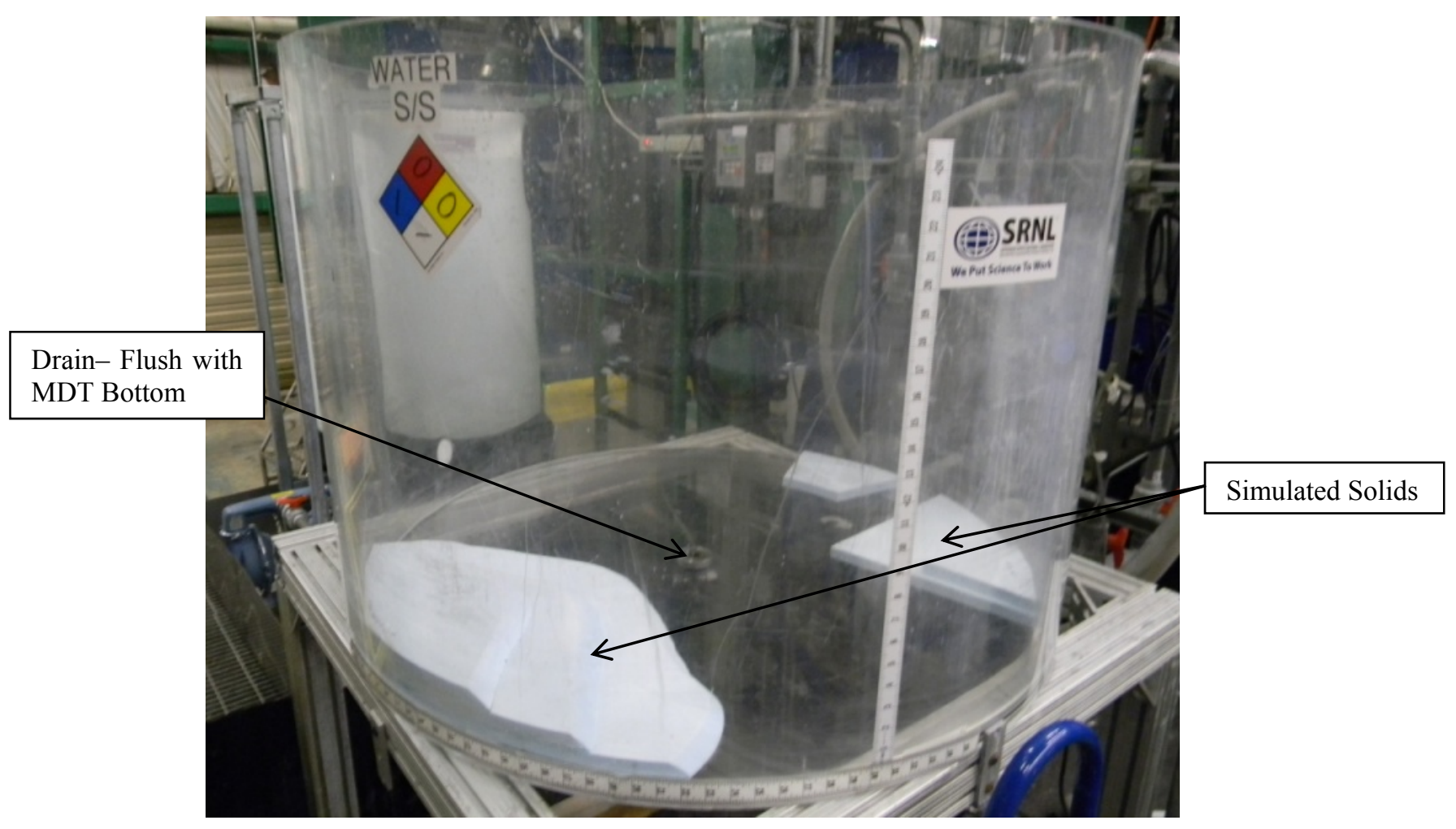

Figure 16. MDT with mock accumulated solids mounds. (Note the new drain in bottom center of the tank to lower the liquid level.)

An important issue with this technique is the contrast between the liquid and the solids to determine the topography of the solids. The camera will have sufficient resolution to accurately define the "shoreline" of the "islands" of solids exposed during the draining process. The cameras can be remotely operated by a laboratory PC controller. A grid or metering gauge (ruler, yardstick, or tape measure) can be attached to the underside of the tank, or possibly floated on the liquid, to help establish scale, which will be done with an empty tank to calibration distances becaus settling solids may obscure the tank bottom from above. Different lighting techniques will be tested to determine which will best distinguish the exposed solids from the submerged solids. Once the photos are taken they can be used to establish a contour map of the solids heel. The volume of the solids can be determined from the contour map. The interstitial volume of the solids can be determined and subtracted from the results.

One bench-top test illustrates the process and accuracy. Yellow sand $\left(320 \mathrm{~mL}\right.$ or $\left.19.5 \mathrm{in}^{3}\right)$ was mounded in a container and enough water added to completely cover the sand, Fig. 17. Water was carefully removed using a suction bulb to lower the water level and overhead photographs were taken at 0.2 " water level increments. In Fig. 17 the water depth equal to 0.8 ", as can be seen on the floating ruler. The left side and bottom side of the shoreline of the sand island are obvious. The other sides are harder to perceive and future tests will be performed to improve the illumination. 


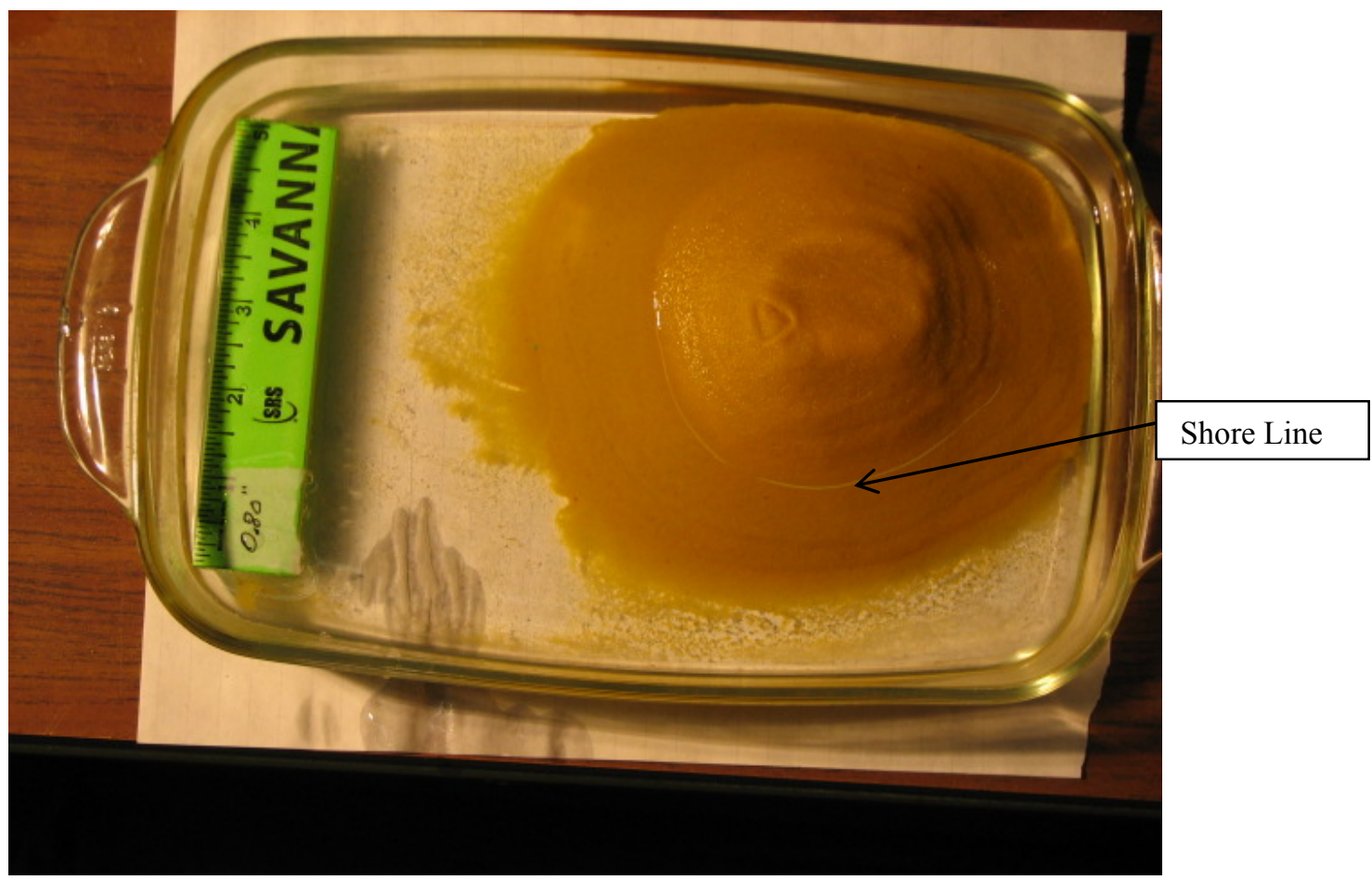

Figure 17. Yellow sand mound in water to determine contrast between liquid and solids, which is can be seen in this photograph

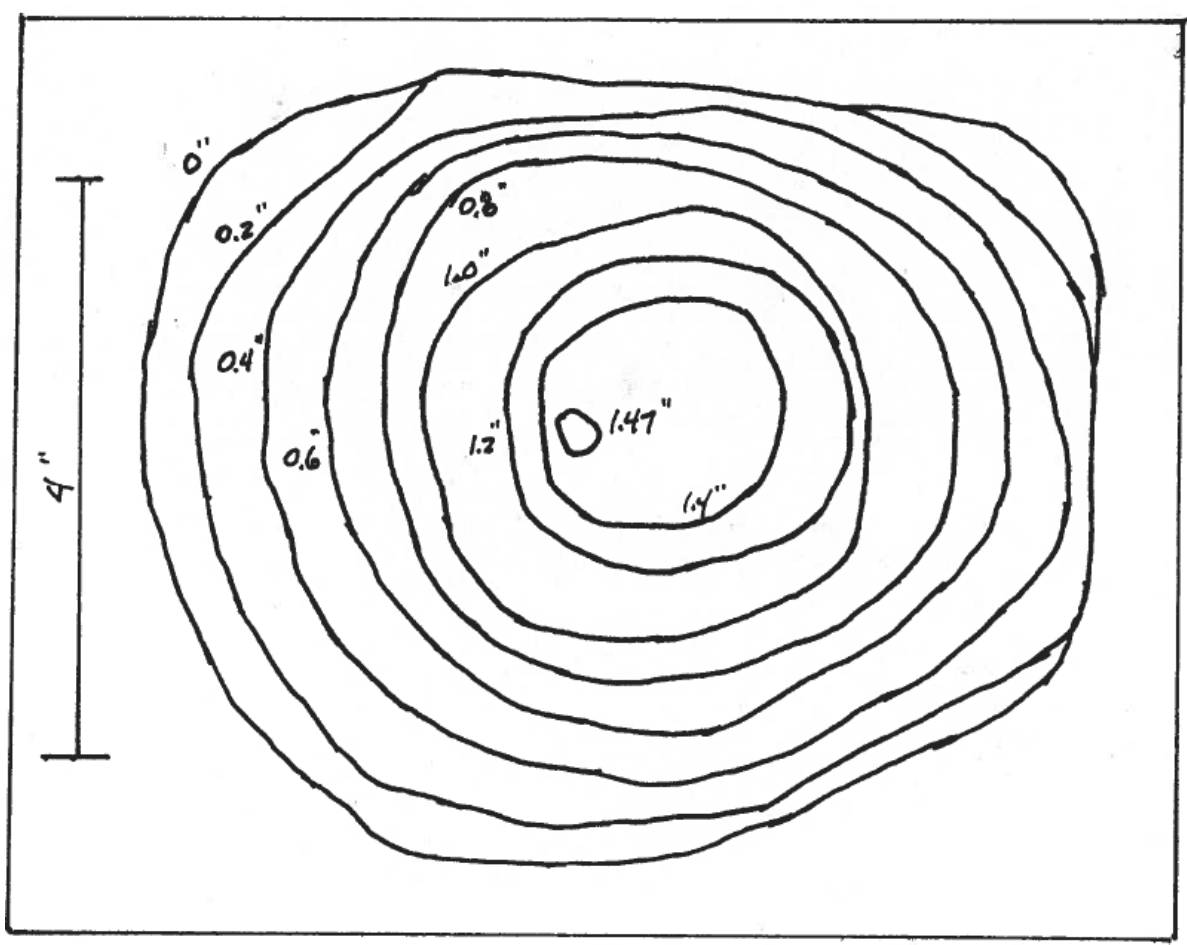

Figure 18. Contour plot of the solids mound shown in the preceding figure 
For each photograph the shoreline between the island and the water was visually determined and manually superimposed on a drawing, Fig. 18. This process of be automated during the full test. The horizontal area of each elevation was measured and volume was computed as the integral of area with respect to elevation. The Simpson's rule [13, p. 171] was used to perform the numerical integration. The resulting volume was $20.1 \mathrm{in}^{3}$, which is within $3 \%$ of the volume of sand originally added. The process was also done using digital images of the photographs and the volume estimated was within $7 \%$ of the known volume. For the actual test, the accumulated solids may be more challenging to map and the remaining gibbsite and zirconium oxide particles will coat the sand that my make discerning the shoreline more difficult. However, with the right lighting this technique is expect to measure the volume to better than $\pm 20 \%$.

\section{$\underline{\text { Secondary Visual-Scanning Method Using Lasers }}$}

Another, more sophisticated method using commercial laser system will be demonstrated at the end of first campaign. It was not included as the principal laser-scanning method to determine the accumulated solids profiles or volume because of the initial estimated cost of $\sim \$ 125 \mathrm{k}$, but a demonstration has been arranged to show the capabilities of this technology so that it can be considered for the entire project. The following is a summary of a visit by SRNL to evaluate the equipment"

On Friday, April 6, 2012 an SRNL representative visited NeoMetrix Technologies, Inc to discuss 3D mapping of the accumulated solids. Dan Perreault, president and CEO of NeoMetrix, demonstrated the 3D mapping capabilities that he thought would best suit the SASS needs. Note, the demonstrated equipment is called Focus ${ }^{3 \mathrm{D}}$, and not the MetraScan ${ }^{\mathrm{TM}}$ that was originally discussed [6]. The hand-held MetraSCAN ${ }^{\mathrm{TM}}$ requires a secondary device, positioned within line of sight to provide a zero reference. It is possible that the MetraSCAN ${ }^{\mathrm{TM}}$ system would be difficult to position because for the device to provide a good reference because of the obstacles such as the rotary pumps and tank walls. Fortunately, the Focus ${ }^{3 \mathrm{D}}$, which is fixed in space to provide 3-D mappings, can provide the same information and accuracy with less hassle and less cost as the MetraSCAN ${ }^{\mathrm{TM}}$. The Focus ${ }^{3 \mathrm{D}}$ has a range of 100 meters and accuracy, at that range, of $\pm 2 \mathrm{~mm}$. Accuracy is improves for smaller ranges. Image accuracy for the MDT tank is expected to be close to $\pm 1 \mathrm{~mm}$.

Neometrix seemed to be a small outfit that sells and provides support for a number of 3D imaging equipment manufacturers, e.g., Creaform makes the MetraSCAN ${ }^{\mathrm{TM}}$ and Faro ${ }_{\circledast}$ makes the Focus ${ }^{3 \mathrm{D}}$. The demonstration consisted of using the Focus ${ }^{3 \mathrm{D}}$ to map a conference room. A hard hat was placed on the table to provide an object that could be analyzed for volume, among other things. The Focus ${ }^{3 \mathrm{D}}$ took data for about 3 minutes and the process is in two steps:

1. A laser scan is made to obtain a point map of the room,

2. A camera scan is made to provide images of the room.

All the data were recorded on a memory chip in the Focus ${ }^{3 \mathrm{D}}$ unit and then the memory card was removed from the Focus ${ }^{3 \mathrm{D}}$ and inserted into a laptop loaded with the requisite software. The software presented a 3D image of the entire room, i.e., the image contained the point map from the lasers and the image taken by the camera. There were several tool bars at the sides and top of the screen that provided manipulation of the image and general image data (orientation, cursor location, etc.). Using the software the image of concern can be isolated, in this case the hard hat, so that a volume could be determined. 
The image of the hard hat was only partial, that is, it was an image of what could be seen by the stationary Focus ${ }^{3 \mathrm{D}}$. The backside and underside of the hard hat was not captured. Those sides not captured can be added by the software to allow the software to provide a volume. These additions are easy to perform with the software. However, the manipulation required a bit of finesse where the contour of the hat created "holes" at the edges of the image. The holes were manually filled in with the software and then a representative volume was determined. For our application the wall and bottom of the tank hidden by the mound will have to be filled in. This will not be a problem since the walls and bottom are simple surfaces.

Note that the image of the hard hat, Fig. 19, appeared rough, or pixilated, when zoomed in on, that is, it didn't appear smooth. The cause was stated to be the shiny surface which will cause minor volumetric errors.

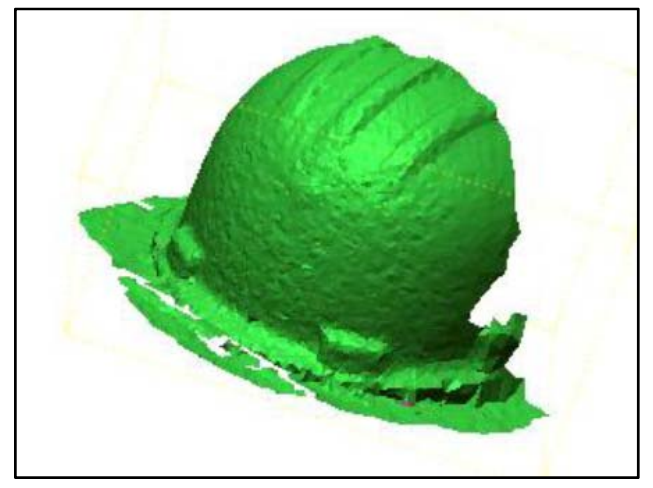

Figure 19. Hard hat image using Focuus 3D

This should not be a problem with the accumulated solids because the surfaces will not be shiny, which would provide a better image. For contrast the Focus ${ }^{3 \mathrm{D}}$ device was zoomed in on the conference room wall (painted a flat color) and the image was much smoother.

During the demonstration the scanning device was attached to a sturdy, camera-style tripod, which the device is setup to receive. For SASS the Focus ${ }^{3 \mathrm{D}}$ device can be attached to a boom that can be positioned over the tank and it can be positioned to capture both of the accumulated mounds or just one mound at a time. The best method of capture can be investigated at the demonstration.

\subsubsection{Stainless Steel Distribution}

A method to determine the distribution of Stainless Steel (SS) particles in the accumulated solids is to sample the solids in several locations throughout the accumulated mounds that can give both the vertical and horizontal locations to the particles. To perform this activity a device was developed that can be placed at a precise location over a mound of solids and collect a core sample with minimal disturbance to the mound.

The concept is capturing a volume with a finger and straw technique, that is, pushing a thin-wall tube into the heel mound, and then sealing off the top of the tube to lift the captured core sample out of the mound. Of course, implementing this simple process without disturbing the mound significantly and doing it at the desired location requires a more sophisticated technique. 
A bench-top test was done by producing a mound of simulant solids then using an actual "straw and finger" to verify a core sample can be removed and retain its integrity. A mound of simulant solids was produced by mixing up a small batch of simulant in a beaker, allowing the solids to settle, then pouring off the supernatant and allowing the solids to drain. Figures 20 and 21 show the results of that test.

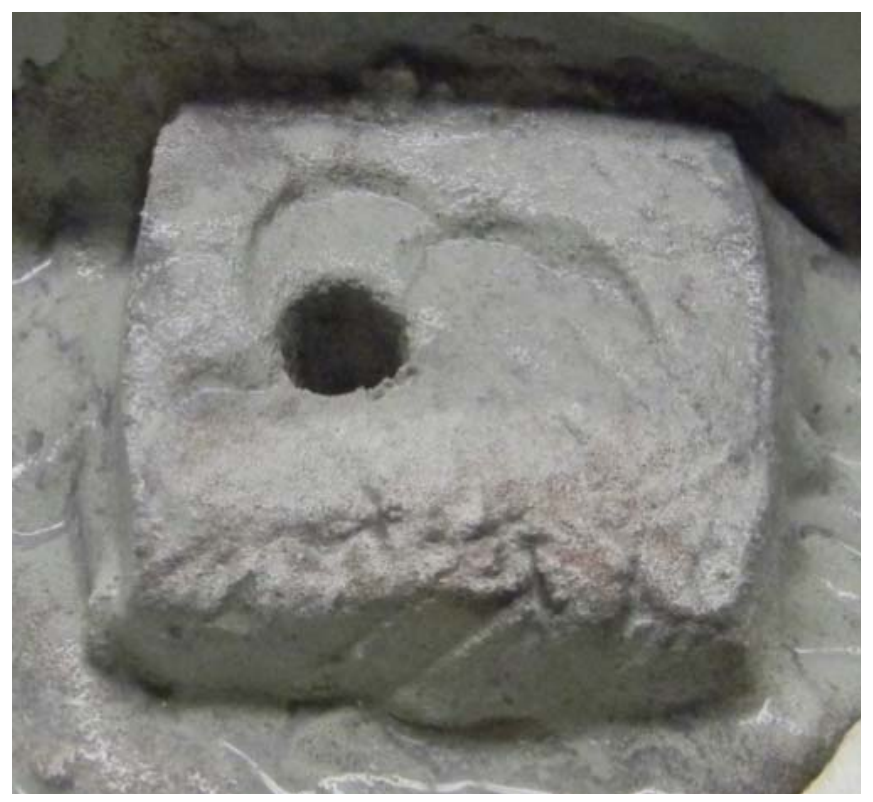

Figure 20. Mound of damp solids: ZrO2, Sand, and Stainless Steel Particles



Figure 21. Core sample drawn from solids shown in the preceding figure

The test was successful and once a core of solids is obtained it will be divided into several sections so the vertical locations of SS particles can be determined. Each section will be placed in a plastic container with liquid and re-suspend. A strong magnet will also be introduced into the container to attract and separate the SS particles. As such, a measure of the vertical distribution of SS particles will be obtained.

With the success of this small test the core sampler, shown as Fig. 22, will be constructed. It incorporates a thin wall outer tube with an O-ring sealed plug, where the tube and seal can move relative to each other. The general operation is simple and involves four steps. 1. The sampler is 
placed at a chosen position, both horizontally and vertically, to sample a solids mound. 2. With the bottom of the sampler tube and the seal plug (the "finger") just touching the very top of the solids to be sampled, the sampler tube is pushed vertically downwards, past the stationary seal plug, into the mound until it reaches the tank bottom, 3. The relative positions of sampler tube and the seal plug are locked. 4. Lift the entire sampler unit with the captured sample of solids out of the support tube. Of course, to actually accomplish these steps properly and accurately the process is more complex.

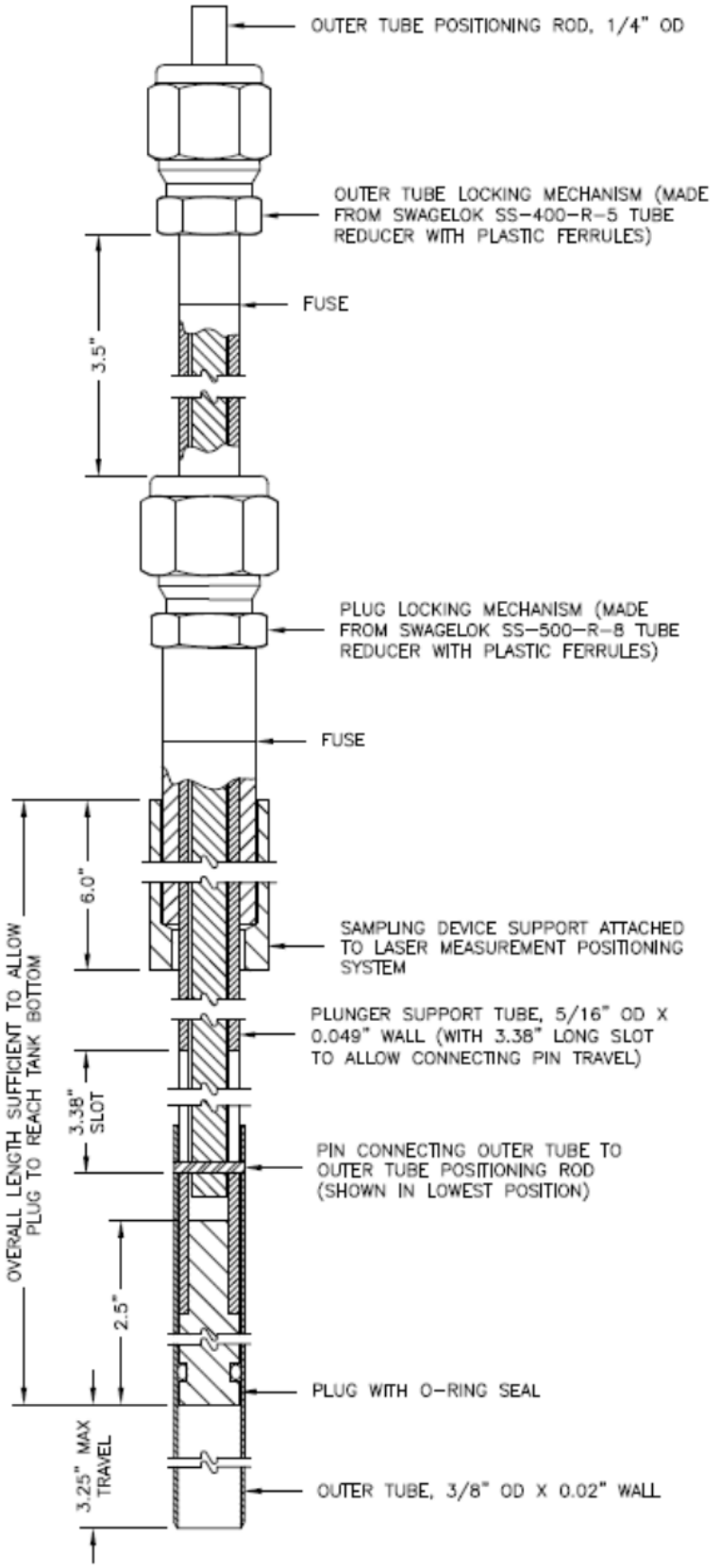

Figure 22. Solids Sampler to Extract a Core of Accumulated Solids 
Note that accumulated solids core samples will only be taken after a full tank transfer, which means the slurry level is at the predetermined heel height of 3.23 inches, i.e., equivalent to the planned 72-inch heel of full scale operation. This heel liquid will be slowly drained from the tank to measure the volume of the accumulated solids as described in Section 2.2.2.2.1. Once the supernatant is fully drained from the heel mounds, the solids core sampler will be put into position to obtain a core sample. The sample locations will be chosen to capture the SS variability within a solids mound.

\subsection{General Sampling}

Throughout the test there will be several points at which simulant sample will be taken to baseline aspects of a test or to archive for future analyses as needed. For instance, the following samples are expected:

- Supernatant before first use

- Slurry for each cycle fill

\subsection{Test Operation}

Refer to Figs. 2 and 3 for the test facility and Table 4 for the campaign volumes

Table 4. Comparison of full scale tank dimensions to those of the MDT

\begin{tabular}{|c|c|c||c|c|c|c|}
\cline { 2 - 7 } \multicolumn{1}{c|}{} & \multicolumn{3}{c||}{\begin{tabular}{c} 
Tank AW-105 \\
\multicolumn{1}{c|}{}
\end{tabular}} & \multicolumn{4}{c|}{ Mixing Demonstration Tank } \\
\cline { 2 - 7 } \multicolumn{1}{c|}{} & gallons Scale & inches & gallons & liters & inches & $\mathrm{cm}$ \\
\hline Tank Inside Diameter & & 900 & & & 40.4 & 102.6 \\
\hline Total Waste & $1,144,000$ & 416 & 103.62 & 392.24 & 18.67 & 47.43 \\
\hline Heel & 198,000 & $72 *$ & 17.93 & 67.89 & 3.23 & 8.21 \\
\hline Total Waste Transfer Volume & 946,000 & 344 & 85.69 & 324.35 & 15.44 & 39.22 \\
\hline Feed Batch & 145,000 & 52.7 & 13.13 & 49.72 & 2.37 & 6.01 \\
\hline Partial Feed Batch & 76,000 & 27.6 & 6.88 & 26.06 & 1.24 & 3.15 \\
\hline
\end{tabular}

*This is the minimum waste height for mixer pump operation in a DST. This height includes as maximum of 70 inches of compacted solids.

\subsection{Overall Operation}

Important Terms (also listed in the Nomenclature):

MDT - Mixing Demonstration Tank - 1/22 scale of the Hanford DST AW-105

Feed Tank (FT) - Tank in which simulant will be prepared and fed to the MDT

Receipt Tank (RT) - There are 7 individual tanks that each will receive one FB transfer

Feed Batch (FB) - Scaled batch of simulant. There are six 13.1-gal and one 6.9-gal batches in each cycle and a heel of $17.9 \mathrm{gal}$ is left in the MDT. 
Cycle $\quad-$ Is one complete fill and empty of the MDT.

MDT Fill - Is 103.6 gal for the first cycle each campaign and 85.7 gal all other cycles.

Campaign $\quad-$ Is the completion of 10 complete cycles done at single jet pump flow rate.

There will be pretest activities that include:

- Shakedown runs to insure that all operates as expected

- Simulant trial test runs, will include*, to determine:

o Remove efficiency of magnetic separator

o Overall operation with receipt tanks and to accumulate solids

o Feed rate to the MDT that will not disturb accumulated solids

o Wait time between each FB to determine effect on accumulated solids

o Functionality and accuracy of laser height measurement

o Accuracy of Volume estimation methods

o Functionality and accuracy of core sampler

o Draining and refilling of MDT heel liquid

o Accumulated Solids Removal

*More tests may be necessary

\subsubsection{Shakedown}

Shakedown runs to insure that all is operational as expected, including:

o Leaks from entire system, e.g, tanks, tubes, valves, pumps

o Proper operation of valves, flow paths, drains, pumps, motors

o Proper operation of instrumentation and confirming accuracies by performing simple measurements, e.g., bucket and stop watch for flow meters

o Calibration of various volumes, e.g., tank full, tank heel, receipt tanks full, etc.

o Proper operation of data acquisition system

o Proper operation of computer controlled activities, e.g., laser positioner

o Prepare simulant, start up, and feed operations

\subsubsection{Trial Test Runs}

The order of the following trial test runs may change due to information learned during each.

\subsubsection{Determine removal efficiency of magnetic separator}

A single FB, currently 13.13 gallons of simulant will be made to test the magnetic separator, see Fig. 8. The batch will be made in the FT, or another tank, from which the entire batch can be drained and sent through the separator at $0.58 \mathrm{gpm}$. This will verify:

0 The effectiveness of separation of the stainless steel particles

0 The ability to transport the simulant through the device

0 The ability, and time, to remove the solids

After the test is finished the batch, with new stainless steel particles, will be reused for the next test.

\subsubsection{Show the overall operation with receipt tanks, magnetic separator, and solids accumulation at scaled flow rates}

This trial is to check the operation of the receipt tank system and to determine jet pump velocities that permit solids to accumulate on the bottom of the tank for Campaign 2. 
From past mixing MDT tests [7] if solids accumulate ${ }^{5}$ at all, at a set jet pump velocity, they occurred during initial tank mixing, before FB transfers begin. Furthermore, there was clear solids accumulations at a jet pump velocity of $22.4 \mathrm{ft} / \mathrm{s} /$ nozzle (or $8 \mathrm{gpm} /$ pump) and a jet pump rotational speed of $1.6 \mathrm{rpm}$; therefore, this combination will be for Campaign 1. However, accumulations at higher velocities/speeds have not been clearly defined. As such a series of jet pump velocities and jet pump rotational speeds will be tested to determine the highest velocity/speed that allows solids to accumulate. The combination of velocity/speed ${ }^{6}$ will be used for Campaign 2. The combinations that will be tried are listed below:

$32.3 \mathrm{ft} / \mathrm{s}$ and a rotation speed of $2.37 \mathrm{rpm}$ (scaling factor exponent ${ }^{7}=1 / 5$ )

[5.76 gpm/nozzle, or $11.5 \mathrm{gpm} / \mathrm{pump} ; 5$ MDT turnovers of a full tank $\left(103.6 \mathrm{gal}^{8}\right) \sim 23 \mathrm{~min}$.]

$27.7 \mathrm{ft} / \mathrm{s}$ and a rotation speed of $2.03 \mathrm{rpm}$ (scaling factor exponent $=1 / 4$ )

[4.94 gpm/nozzle, or $9.9 \mathrm{gpm} /$ pump; 5 MDT turnovers of a full tank (103.6 gal.) $26 \mathrm{~min}$.]

$24.4 \mathrm{ft} / \mathrm{s}$ and a rotation speed of $1.79 \mathrm{rpm}$ (scaling factor exponent $=7 / 24$ )

[4.35gpm/nozzle, or $8.7 \mathrm{gpm} / \mathrm{pump} ; 5 \mathrm{MDT}$ turnovers of a full tank (103.6 gal.) $30 \mathrm{~min}$.]

$22.4 \mathrm{ft} / \mathrm{s}$ and a rotation speed of $1.57 \mathrm{rpm}$ (scaling factor exponent $=1 / 3$ )

[4.00 gpm/nozzle, or $8 \mathrm{gpm} /$ pump; 5 MDT turnovers of a full tank (103.6 gal.) $32 \mathrm{~min}$.]

Operation: With a full batch of simulant the MDT will be filled at a predetermined rate, e.g., $2 \mathrm{gpm}$ to observe the overall operation. The full MDT will have most of the solids sitting on the tank bottom before mixing begins. Once the MDT is full the jet pumps will be turned to pick up the solids and mix the MDT at the first jet pump velocity and rotational speed listed above. After waiting for approximately 5 MDT turnovers the receipt tanks will be filled one at a time at the planned rate of $0.58 \mathrm{gpm}$. During a transfer the magnetic separator will be used to separate the stainless steel particles to determine the batch-to-batch operation of the separator. After filling of Receipt Tank 1 with the first FB, shut the MDT mixing and empty the magnetic separator of SS solids to be measured. As soon as the magnetic separator is ready. Repeat the entire FB transfer process once more for the first jet pump/rotational speed combination filling Receipt Tank 2. If solids accumulate this will be the combination used for Campaign 2. If solids do not accumulate then repeat the process above to fill Receipt Tanks 3 and 4 using the second jet pump velocity and rotational speed combinations and then again for the third combination for Receipt Tanks 5 and 6 . However, whichever is the first combination to allow solids to accumulate, the remaining receipt tanks shall be filled using the Campaign 1, last combination, to prepare the MDT for the next trial: 3.1.2.3. Once the MDT volume is reduced to the heel height, i.e., 3.23 inches, the jet pumps will be stopped. The settled solids in the receipt tanks will be noted and then the contents of all the tanks will be returned to the MDT feed tank.

\subsubsection{Feed rate to the MDT that will not disturb accumulated solids}

After waiting 24 hours for solids in the 3.23-inch heel to settle the tank will be refilled, with no mixing, which is prototypical, at a rate with does not disturb the solids. The fill

\footnotetext{
${ }_{6}^{5}$ Actually the solids are more being picked up and redistributed in poorly mixed regions.

${ }^{6}$ As explained in Ref. 3, the scaling method uses exponents based the model criteria and for this task the chosen exponent, i.e., $1 / 3,1 / 4,1 / 5$, etc. is used to determine both the scaled jet pump velocity and jet pump rotational speed. That is, whatever exponent is chosen it determined both variables, which make them dependent.

${ }^{7}$ Scale factor exponents and scaling models are explained in Ref. 3. Note the MDT at an inside diameter of 40.4 in., Table 4, and full size AW-105 tank at 900 in. is linearly scaled at 40.4/900 =1/22.

${ }^{8}$ Note, as the MDT volume is reduced after each transfer from $103.6>90.5>77.3>64.2>51.1>38.0>24.8>17.9$ gal, then the time to attain 5 MDT turnovers successively drops. The proper time for each situation will be determined before each mixing.
} 
rate will begin at $2 \mathrm{gpm}$ and observed. If those mounds of solids are visually disturbed, as seen on the bottom and sides of the mounds, then the rate will be reduced until the mounds appear unaffected. This will be the feed rate used throughout the test. The filling will continue until the MDT tank is at the full mark.

\subsubsection{Wait time between each FB to determine effect on accumulated solids ${ }^{9}$}

After the MDT is full this trial is ready to begin. Three transfers will be done to observe the pattern of the settling solids, then longer intervals will be used to observe differences in accumulated solids:

1. Turn on the jet pumps, using the $8 \mathrm{gpm}$ flow rate/pump (4 gpm/nozzle, or $22.4 \mathrm{ft} / \mathrm{s}$ nozzle velocity) and wait for 5 MDT turnovers, approximately 30 minutes. Transfer a FB transfer, which takes approximately 23 minutes. The MDT mixing will be stopped and the solids in the first RT will be allowed to settle for approximately 20 minutes, after the wait time any changes to the settled solids will be noted.

2. Step 1 is repeated with the second RT.

3. Step 1 is repeated with the third RT.

When the third RT is full the MDT will shut down and allowed to sit for 2 or 3 hours.

4. Step 1 is repeated with the fourth RT.

When the fourth RT is full the MDT will shut down and allowed to sit for at least 12 hours.

5. Step 1 is repeated with the fifth RT.

6. Step 1 is now repeated two more times with only the 20 wait time to fill RTs six and seven, leaving the 3.23-inch heel in the tank.

\subsubsection{Determine functionality and accuracy of laser height measurement}

The distance lasers probes are set into position and the computer will be used to both position the probe and record the data. The time of operation will be determined and the probes' ability to measure through the slightly opaque liquid will be evaluated. If accuracy is compromised by suspended solids and the time to establish a signal takes too long this operation will be redone after the solids are exposed. The operation of the probe positioner will be tested and the data obtained will be used to set up the process of converting the $3 \mathrm{D}$ points to topographical and volume measurements.

\subsubsection{Determine accuracy of Volume estimation methods}

There are two methods plan: Liquid displaced and 3-D topographical photographs

1. The procedure and accuracy of the volume estimation through the measurement of displaced liquid volume will be checked, e.g., draining liquid, measuring liquid level, choose level increments to measure.

2. Once the solids are full exposed the 3-D camera system will be tested to obtain topographical pictures of the MDT bottom and to estimate the volume of the settled solids.

\subsubsection{Determine functionality and of core sampler and its ability to capture a complete core}

The core sampler will now be used by setting it in the positioner system to take core samples of the solids in several locations to judge the operation. The removed core hole will be checked visually to estimate the sampler's ability to capture an entire column of solids from the sample location.

\footnotetext{
${ }^{9}$ The sequence and steps of 3.1.2.4 are only an example. Both may vary depending on observations.
} 


\subsubsection{Refill liquid to reestablish MDT heel}

Unless a campaign is at its end the heel liquid removed to measure the accumulated solids needs to be replaced. This will be checked to determine the rate of refill that does not disturb the solids, if it can put through the bottom or it needs to be put in through the top, if the drain valve becomes plugged during the refill, etc.

\subsubsection{Accumulated Solids Removal}

The very last measurement in the MDT of each campaign will be to collect the accumulated solids to obtain a measure of the mass of stainless steel remaining to complete a mass balance. This pretest activity will remove the remaining accumulated solids to obtain the stainless steel particles in the settled solids. A mechanical method may be tried to remove each of the two mounds separately so the particle concentration in each is obtained. However, if it not possible that supernatant will be added to suspend solids to be sluiced out of the tank to obtain the concentration of from all the settled solids.

\subsubsection{Planned Test Operation}

\subsubsection{Campaigns 1 \& 2}

Notes: For Campaign 1: MDT Jet Pumps set at 8 gpm/pump or 4 gpm.nozzle $(22.4 \mathrm{ft} / \mathrm{s})$ For Campaign 2: MDT Jet Pumps set at the rate determined from Step 3.1.2.2 ${ }^{10}$ Ten complete Fill and Empty Cycles at on Pump Jet Energy Level

1. Fill ${ }^{11}$ the FT with the components of simulated tank farm waste: 103.6 gal., heel included or 85.7 gal. when the MDT already filled with a heel.

2. Transfer the prepared Cycle batch to MDT, without jet pumps operating at the rate determined from Section 3.1.2.3. Take a $25 \mathrm{ml}$ sample from the transfer line and store.

3. Start the jet pumps to mix the MDT and continue mixing until simulant is stable in color, slurry movement, and bottom solids. At least 5 MDT turnovers, which is approximately 30 minutes. Pump operation starts by first initiating pump rotation by synchronizing the will be started.

4. Transfer a FB: 13.13 gal., to the next empty RT at $0.58 \mathrm{gpm}$. The volume is determined by the full line on the RT and will take approximately $23 \mathrm{~min}$.

5. Stop the MDT jet pumps.

6. Wait 20 minutes for solids in RT to settle, then note the settled heights at the time.

7. Note observations, take pictures, remove stainless steel particles from separator and measure and record mass of particles. Replace separator in place

8. When the wait time determined in Section 3.1.2.4 has elapsed the next transfer is ready.

9. Repeat Steps 3 to 8 five (5) times.

\footnotetext{
${ }^{10}$ The largest jet pump flow rate that allows solids to accumulate will be used for Campaign 2. If in Step 3.1.2.2 no solids accumulated at any velocity above the Campaign 1 velocity of $22.4 \mathrm{ft} / \mathrm{s} / \mathrm{nozzle}$ than Campaign 2 shall be terminated.

${ }^{11}$ The FT is supplied with new simulant for the first and second cycles. At the end of the first cycle the seven RTs will be emptied into two 55-gal settling drums. At the end of the second cycle the seven RTs will be emptied into another set of two 55-gal settling drums and the first two drums will be decanted and transferred to the FT through a bag filter to reuse the supernatant to make up the third MDT fill. This process will continue until the end of Campaign 1 after which the process will be repeated.
} 
10. When sixth FB is processed then repeat Steps 3 to 9 once more but only transfer 6.9 gal. to the seven RT, which should leave a simulant heel of 3.23 inches in the MDT.

This completes one complete fill and empty cycle

11. Repeat Steps 1 through 10 nine more times to complete 10 full cycles and Campaign 1. However, at the end of the $1^{\text {st }}, 5^{\text {th }}$, and $10^{\text {th }}$ cycle the volume of the accumulated solids will be measured. Go to the next section to measure accumulated solids.

12. Campaign 2 - Repeat Steps 1 to 11 at the higher jet pump velocity

\subsubsection{Accumulated Solids Measurement}

Within each campaign, at the end of the $1^{\text {st }}, 5^{\text {th }}$, and $10^{\text {th }}$ cycles, the accumulated solids will be measured. At this point the MDT simulant level will be at the heel height, i.e., 3.23 inches, to begin the accumulated solids measurement sequence:

1. Laser height measurement

(If in Section 3.1.2.5 this method was determined to not operate accurately because it takes too long for solids to settle to allow the lasers to penetrate the liquid, it will be done after the heel liquid is drained and the solids mounds exposed.)

Two or more distance laser meters will be placed in the probe positioner, Fig. 12, and the computer will be set to scan the accumulated solids. The plan is that the computer will move the laser probes the every point in Fig. 12, in approximately 30 minutes to map both areas. The information will be used to develop a topographical map of the mounds and the area around the mounds.

2. Volume Determination - Liquid displaced and Topographical Mapping

During this procedure the heel liquid will be drained and then replaced after core samples are taken from the mounds of accumulated solids. A drain installed in the MDT will be used to lower the liquid level. This drain is flush with the bottom of the tank so it will not case interference during mixing and it will only opened after a time determined in Section 3.1.2.6 to allow most of the solids to settle and minimize the solids that will drain during this procedure. The steps will be:

a. Wait until solids have settled.

b. Set up the camera system on top of the MDT.

c. Open drain to fill manometer to measure height.

d. Drain enough liquid for the simulant level to just match the highest point of solids.

e. Lower the liquid level by the amount determined in Section 3.1.2.6 and measure the displaced volume by measuring the volume of the liquid removed.

f. Adjust the lighting to best contrast the liquid-solid interface on the solids mounds for the topographical mapping. Take the appropriate photographs.

g. Repeat the previous two steps until the entire height of settle solids are exposed and the liquid drained from the MDT.

3. Core Sampling

Set the core sampler into the probe positioner to begin the core sampling. The shape of the settled solids will be used to guide where core sample are to be take, but several same will be taken at both low and high locations to obtain a good representation of the location of the stainless steel particles in the mounds. Only one mound will be chosen for 
sample for the $1^{\text {st }}$ and $5^{\text {th }}$ cycle samples. Care must be taken to minimize the disturbance to the mound being sampled. The basic steps of sampling will be:

a. Place sampler in probe position

b. Have computer place probe at the desired location. These locations will be chosen to best measure the distribution of SS particles, i.e., low, medium and high portions of a mound, but it is also important to not destroy the mound in the process; therefore, specific location cannot be chosen a priori.

c. Extract a core of solids

d. Remove sampler from positioner and remove sample.

e. Segment the core sample in several pieces, making sure two note the location of each segment from top to bottom.

f. Place each segment into a container with liquid to suspend the solids and then use a magnet to separate the solids from the segment sample.

g. Measure and record mass of stainless steel particles.

h. Repeat Steps a to $g$ for each location until the mound is sampled as planned.

i. After all samples are obtained, then the collected heel liquid is returned to the MDT to reestablish the heel so the next test cycle can be performed. Return to the overall test campaign to perform the next cycle, unless this is the $10^{\text {th }}$ cycle at which case the next Step is performed.

\section{Tenth Cycle Measurements}

a. Core Sampling - At the end of each campaign and the end of the $10^{\text {th }}$ cycle the core sampling will be performed as listed in Step 3; however, at the end of the campaign both solids mounds will be sampled. They will be sampled in similar locations to compare the variation of stainless steel particle in both mounds.

b. Accumulated Solids Removal - Finally, the very last measurement in the MDT of each campaign will be to collect the accumulated solids to obtain a measure of the mass of stainless steel remaining to complete a mass balance. During pretesting the method of this collection will be perfected. It will be either adding supernatant to suspend the solids to be sluiced out or each mound may be removed mechanically so a measure of the stainless steel particles in each mound can be measured.

\subsection{Measurements}

Of course, throughout the test program many measurements will be made. The goal is to make all measurements that meet the measurement uncertainty requirement of equal to better than $\pm 20 \%$ as stated in Lee [3; Section 3.4.1.40]. The data will be recorded either manually, e.g. Lab Temperatures, Lab Pressures, Liquid Heights, etc. in the laboratory notebook [14] or electronically by the data acquisition system, e.g., Flow Rates, Rotational Speeds, etc. or both, e.g., analytical results. The following is the type measurements expected:

\subsubsection{Flow}

There will be at least four (4) flow rates measured during the test: Flow rate to fill MDT, Flow rate to the two jet pumps, and the flow rate out of the MDT. These will be done with installed magnetic flow meters and have calibrated uncertainties of approximately $1 \%$.

\subsubsection{Concentrations (Mass)}

Generally, concentrations are determined by measuring the mass of a constituent, which can be performed very accurately, i.e., $<1 \%$. This is expected with the measure of the stainless steel 
particles. However, when estimating concentrations from a series of measurements, e.g., the concentration of stainless steel particles in accumulated solids that will depend on many cumulative measurements the uncertainty will increase, but it is still expected to be considerably less that $\pm 20 \%$. Other concentrations that are estimated by volume, e.g., settled sand, may have larger uncertainties and will be deemed only qualitative.

\subsubsection{Linear Dimensions}

The laser technique will measure the height, width, and length of the mounds, as well as point to point distances. The positioner uncertainties are on the order of $1 \%$ from one point to the next. The positioner can place a probe along a radius every 0.75 inch $(19.1 \mathrm{~mm})$ with a position uncertainty of $\pm 0.1 \mathrm{~mm}$. The overall uncertainty of a mound from one end to another should also be less that $1 \%$. The positioner can place a probe every $3^{\circ}$ of angle of arc and with uncertainty of $\pm 0.01^{\circ}$ then point to point uncertainty is less than $1 \%$.

\subsubsection{Volumes}

This measurement is expected to have the largest uncertainty, but it expected to be better that the required $\pm 20 \%$. The volume of accumulated solids will be estimated by more than one method, e.g., liquid displaced, topographical, etc. Initial volume displacement tests with known volumes and controlled situations gave results of better than $\pm 3 \%$; therefore, the goal of attaining accurate volume estimations appears achievable.

\subsubsection{Miscellaneous}

Several samples will be taken to baseline or confirm expectation. For example, the supernatant will be sampled once mixed from stored totes to baseline the material that will suspend the solids. Samples have already been taken of all the solids to be tested and the particle size distributions are shown in App. A.

\subsubsection{Archive}

Samples will be taken as needed and archived to be analyzed if the project deems it necessary. For example, sample of each MDT fill simulant, possibly of the individual receipt tanks, to confirm concentrations, etc.

\subsection{Post Test Activities}

1. Transmit Preliminary date to WRPS on or before 7 July 2012.

2. Analyzed all test data.

3. Transmit the draft final report on or before 3 August 2012.

4. Disposition all the processed simulant

\subsection{References}

1. Inter-Entity Work Order, 2012, "Solids Accumulation Scouting Studies," Work Order No. M0SRV00070, January 31.

2. Duignan, M.R., Steeper, T.J., Steimke, J.L., Leishear, R.A., 2012. "Solids Accumulation Scouting Studies," Task Technical and Quality Assurance Plan, Doc. No. SRNL-RP-2012-00057. 
3. Lee, K. P., 2012. "Waste Feed Delivery Mixing and Sampling Program Limits of Performance and Solids Accumulation Scouting Studies Test Plan," Doc. No. RPPPLAN-52005, Rev. 0

4. Thien, M.G., Sexton, R.A., 2012. "Waste Feed Delivery Mixing and Sampling Program Plan and Test Requirements," RPP-PLAN-41807, Rev. 1.

5. Lee, K.P., B.E. Wells, P.A. Gauglitz, and R.A. Sexton, 2012. "Waste Feed Delivery Mixing and Sampling Program Simulant Definition for Tank Farm Performance Testing," RPP-PLAN-51625, Rev.0.

6. Duignan, M.R., Steeper, T.J., Steimke, J.L., Fowley, M.D., 2012. "Simulant Development and Sampling Plan." Memorandum No. SRNL-L3100-2012-00024, Rev. 0 .

7. Adamson, D.J., Poirier, M.R., Steeper, T.J. 2009. "Demonstration of Simulated Waste Transfers from Tank AY-102 to the Hanford Waste Treatment Facility," Doc. No. SRNL-STI-2009-00717, Rev. 0.

8. Adamson, D.J., Restivo, M.L., Steeper, T.J., Greer, D.A. 2010. "Demonstration of Mixer Jet Pump Rotational Sensitivity on Mixing and Transfers of the AY-102 Tank," Doc. No. SRNL-STI-2010-00521, Rev. 0.

9. Adamson, D.J., Gauglitz, P.A. 2011. "Demonstration of Mixer and Transferring Settling Cohesive Slurry Simulants in the AY-102 Tank," Doc. No. SRNL-STI-201100278, Rev. 0.

10. Herting, D.L. to Nelson, E.A., 2007. "Preparation of Simulated SST Early Feed Solution for Pilot Plant," Doc. No. CH2M-0701541.

11. Dullien, F.A.L. 1992. Porous Media - Fluid Transport and Pore Structure, $2^{\text {nd }}$ Ed. Academic Press, Inc.

12. A. Dale Marzolf, Matt Folsom, 2010, “3-D Mapping Technologies for High Level Waste Tanks," Doc. No. SRNL-TR-2010-00239, Rev. 0.

13. Kaplan, W., 1952. Advanced Calculus, Addison-Wesley Pub. Co.

14. Duignan, M.R., 2012, "Solids Accumulation Scouting Studies," SRNL Notebook No. SRNL-NB-2012-00033. 


\title{
Appendix A
}

Particle Size Distribution of Undissolved Solids for the Simulated Tank Waste

\author{
Gibbite \\ Zirconium Oxide \\ Sand \\ $\&$
}

Stainless Steel (with and without sonication) 
SRNL-STI-2012-00239

Revision 0

\section{PSD for Gibbsite in $\mathrm{H} 2 \mathrm{O}$ and with no sonication}

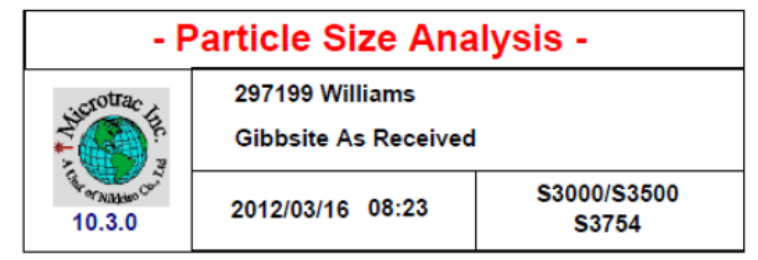

\begin{tabular}{|c|c|}
\hline Data Item & Value \\
\hline MV(um): & 11.4 \\
\hline MN(um): & 0.778 \\
\hline MA(um): & 4.31 \\
\hline CS: & 1.391 \\
\hline SD: & 8.16 \\
\hline & \\
\hline
\end{tabular}

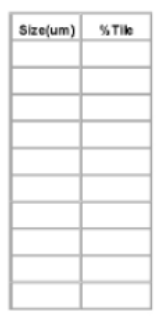

\begin{tabular}{|c|c|}
\hline \%Tile & Size(um) \\
\hline 10.00 & 1.750 \\
\hline 20.00 & 3.51 \\
\hline 25.00 & 4.41 \\
\hline 40.00 & 7.44 \\
\hline 50.00 & 9.84 \\
\hline 60.00 & 12.22 \\
\hline 70.00 & 14.68 \\
\hline 75.00 & 16.04 \\
\hline 90.00 & 22.34 \\
\hline 95.00 & 27.68 \\
\hline
\end{tabular}
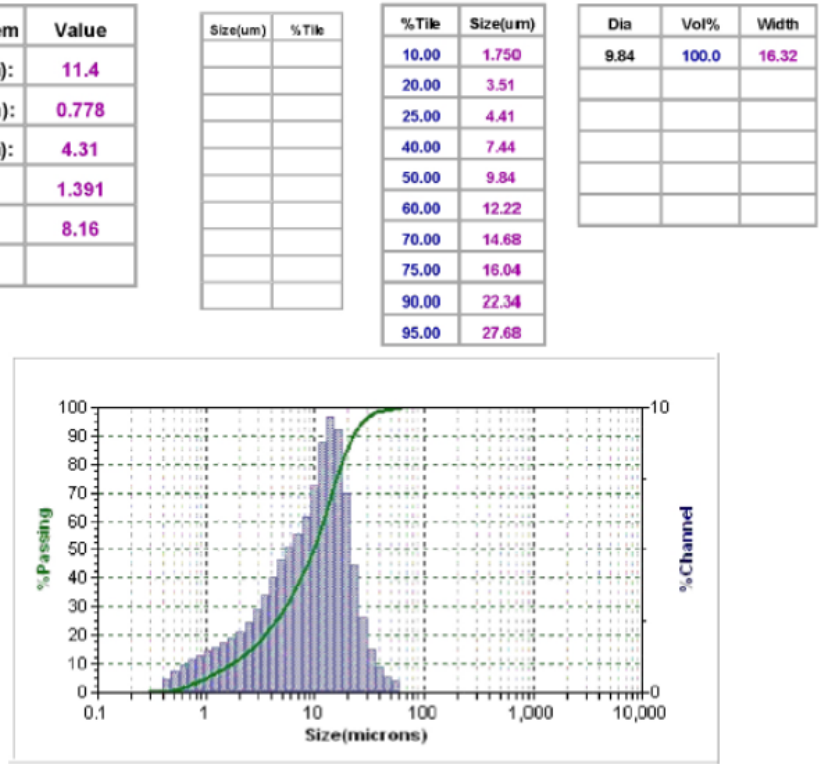

in DiH2O

\begin{tabular}{|c|c|c|c|c|c|c|c|}
\hline Distritubon: & Volume & Run nme: & $30 \mathrm{sec}$ & Fluid: & WaTER & & \\
\hline Propession & Standard & Rum Num: & Arg of 3 & Flad Ret, index & 1.33 & Laading Factor: & 0.039 \\
\hline Upper Edage: & 1008 & Paracle: & $\begin{array}{c}\text { NST } 1634 \text { STD } \\
\text { 10.155.07A }\end{array}$ & Abew Residuat: & 0 & Trantabsesion: & 0.91 \\
\hline Lower Edoe: & 0.243 & Transparmency. & Aosoloting & Below Reviduat: & 0 & RMS Residaut: & SBrEes \\
\hline Residulat: & Enabted & Part Ret. index: & Na & & & Flow & $50 \%$ \\
\hline Mum, Charmis: & so & Part snape: & inreguar & Cotlio: & 0695 & Usoric Pouer: & Na \\
\hline Analyois Mode: & 52000 & & & & & Useric Time: & NA \\
\hline Fater: & Enabted & DB Recerd: & 9030 & Recale sianus: & & Serial Mum: & $\sin \omega$ \\
\hline Andysis Gain: & Defadt & & & & & tMoe & \\
\hline
\end{tabular}

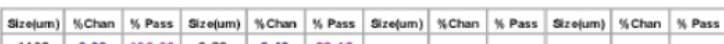
\begin{tabular}{|c|c|c|c|c|c|}
\hline 1408 & 0.00 & 100.00 & 3.89 & 3.43 & 22.12 \\
\hline 1184 & 0.00 & 100.00 & 3.28 & 289 & 18.69 \\
\hline
\end{tabular}

\begin{tabular}{|l|l|l|l|l|l|}
\hline 1184 & 0.00 & 100.00 & 3.27 & 2.89 & 18.59 \\
\hline
\end{tabular}

\begin{tabular}{|l|l|l|l|l|l|l|}
\hline $\mathbf{9 9 5 . 6}$ & $\mathbf{0 . 0 0}$ & 100.00 & $\mathbf{2 . 7 5 0}$ & $\mathbf{2 . 4 7}$ & 15.80 \\
\hline $\mathbf{8 5 7 2}$ & 0.00 & 100.00 & 2.312 & 2.15 & 13.33 \\
\hline
\end{tabular}

\begin{tabular}{|l|l|l|l|l|l|l|}
\hline 837.2 & 0.00 & 100.00 & 2.312 & 2.15 & 13.33 \\
\hline 72.0 & 0.00 & 10.00 & 1.945 & 1.90 & 11.13 \\
\hline
\end{tabular}

\begin{tabular}{|l|l|l|l|l|l|l|}
\hline 704.0 & 0.00 & 100.00 & 1.945 & 1.90 & 11.18 \\
\hline
\end{tabular}

\begin{tabular}{|l|l|l|l|l|l|}
\hline 592.0 & $\mathbf{0 . 0 0}$ & 100.00 & $\mathbf{1 . 6 3 5}$ & $\mathbf{1 . 7 1}$ & $\mathbf{9 . 2 8}$ \\
\hline 497.3 & 0.00 & 100.00 & $\mathbf{1 . 3 7 5}$ & 1.56 & 7.55 \\
\hline
\end{tabular}

\begin{tabular}{|l|l|l|l|l|l|}
\hline 497.8 & 0.00 & 100.00 & $\mathbf{1 . 3 7 5}$ & 1.56 & 7.57 \\
\hline $\mathbf{4 1 8 . 6}$ & 0.00 & 100.00 & $\mathbf{1} 158$ & 1.43 & $\mathbf{6 0 1}$ \\
\hline
\end{tabular}

\begin{tabular}{|l|l|l|l|l|l|}
\hline $\mathbf{4 1 8 . 6}$ & $\mathbf{0 . 0 0}$ & 100.00 & $\mathbf{1 . 1 5 6}$ & $\mathbf{1 . 4 3}$ & $\mathbf{6 . 0 1}$ \\
\hline 3520 & 0.00 & 100.00 & 0.972 & 1.30 & 4.50 \\
\hline
\end{tabular}

\begin{tabular}{|c|c|c|c|c|c|c|}
\hline 296.0 & 0.00 & 100.00 & 0.818 & 1.15 & 3.28 \\
\hline
\end{tabular}

\begin{tabular}{|l|l|l|l|l|l|}
\hline 296.0 & 0.00 & 100.00 & 0.818 & 1.15 & 3.28 \\
\hline 248.9 & 0.00 & 100.00 & 0.688 & 0.96 & 2.13 \\
\hline
\end{tabular}

\begin{tabular}{|l|l|l|l|l|l|}
\hline 248.9 & 0.00 & 100.00 & 0.688 & 0.96 & 2.13 \\
\hline 209.3 & 0.00 & 100.00 & 0.578 & 0.71 & 1.17 \\
\hline
\end{tabular}

\begin{tabular}{|l|l|l|l|l|l|}
\hline 209.3 & 0.00 & 100.00 & 0.578 & 0.71 & 1.17 \\
\hline 176.0 & 0.00 & 100.00 & 0.486 & 0.46 & 0.46 \\
\hline 108.0 & 0.00 & 100.00 & 0.409 & 0.00 & 0.00 \\
\hline
\end{tabular}

\begin{tabular}{|l|l|l|l|l|l|}
\hline 176.0 & 0.00 & 100.00 & 0.486 & 0.46 & 0.46 \\
\hline 148.0 & 0.00 & 100.00 & 0.409 & 0.00 & 0.00 \\
\hline 124.5 & 0.00 & 10.00 & 0.394 & 0.00 & 0.00 \\
\hline
\end{tabular}

\begin{tabular}{|l|l|l|l|l|l|}
\hline 148.0 & 0.00 & 100.00 & 0.409 & 0.00 & 0.00 \\
\hline 124.5 & 0.00 & 100.00 & 0.344 & 0.00 & 0.00 \\
\hline
\end{tabular}

\begin{tabular}{|l|l|l|l|l|l|l|}
\hline 104.7 & 0.00 & 100.00 & 0.2890 & 0.00 & 0.00 \\
\hline
\end{tabular}

\begin{tabular}{|c|c|c|c|}
\hline 88.00 & 0.00 & 100.00 \\
\hline
\end{tabular}

\begin{tabular}{|l|l|l|}
\hline 88.00 & 0.00 & 100.00 \\
\hline 74.00 & 0.00 & 100.00 \\
\hline 6.23 & 0.39 & 100.00
\end{tabular}

\begin{tabular}{|l|l|l|}
\hline 74.00 & 0.00 & 100.00 \\
\hline 62.23 & 0.39 & 100.00 \\
\hline 5233 & 0.58 & 99.61 \\
\hline
\end{tabular}

\begin{tabular}{|c|c|c|}
\hline 62.23 & 0.39 & 100.00 \\
\hline 52.33 & 0.58 & 99.51 \\
\hline 44.00 & 0.91 & 99.03 \\
\hline
\end{tabular}

\begin{tabular}{|l|l|l|}
\hline 52.33 & 0.58 & 99.61 \\
\hline 44.00 & 0.91 & 99.03 \\
\hline
\end{tabular}

\begin{tabular}{|l|l|l|}
\hline 37.00 & 1.51 & 98.12 \\
\hline
\end{tabular}

\begin{tabular}{|l|l|l|}
\hline 31.11 & 2.61 & 96.61 \\
\hline
\end{tabular}

\begin{tabular}{|l|l|l|}
\hline 31.11 & 2.61 & 96.61 \\
\hline 26.16 & 4.47 & 94.00 \\
\hline 22.00 & 7.00 & 09.53 \\
\hline
\end{tabular}

\begin{tabular}{|l|l|l|}
\hline 26.16 & 4.47 & 94.00 \\
\hline 22.00 & 7.00 & 89.53 \\
\hline
\end{tabular}

\begin{tabular}{|l|l|l|}
\hline 22.00 & 7.00 & 89.53 \\
\hline 18.50 & 9.22 & 82.53 \\
\hline 15.56 & 9.69 & 73.31 \\
\hline
\end{tabular}

\begin{tabular}{|l|l|l|}
\hline 18.50 & 9.22 & 82.53 \\
\hline 15.56 & 9.69 & 73.31 \\
\hline 13.08 & 8.77 & 63.62 \\
\hline
\end{tabular}

\begin{tabular}{|c|c|c|}
\hline 13.08 & 8.77 & 63.62 \\
\hline 11.00
\end{tabular}

\begin{tabular}{|l|l|l|}
\hline 11.00 & 7.26 & 54.85 \\
\hline 9.25 & 0.14 & 4.59 \\
\hline
\end{tabular}

\begin{tabular}{|l|l|l|l|}
\hline 9.25 & 6.14 & 47.59 \\
\hline 7.28 & 5.52 & 4.05 \\
\hline
\end{tabular}

\begin{tabular}{|l|l|l|}
\hline 7.78 & 5.52 & 41.45 \\
\hline 6.54 & 5.11 & 35.93 \\
\hline
\end{tabular}

\begin{tabular}{|l|l|l|}
\hline 6.54 & 5.11 & 35.93 \\
\hline 5.50 & 4.55 & 30.2 \\
\hline
\end{tabular}

\begin{tabular}{|l|l|l|}
\hline 5.50 & 4.65 & 30.32 \\
\hline 4.62 & 4.05 & 25.17 \\
\hline
\end{tabular}

\begin{tabular}{|l|l|l|}
\hline 4.62 & 4.05 & 26.17 \\
\hline
\end{tabular}

Warnings: NONE 


\section{PSD for Zirconium Oxide in $\mathrm{H} 2 \mathrm{O}$ and with no sonication}

\begin{tabular}{|c|c|c|}
\hline \multicolumn{3}{|c|}{ - Particle Size Analysis - } \\
\hline & 297198 Williams & \\
\hline & ZrO2 As Received & \\
\hline 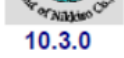 & 2012/03/26 07:53 & $\begin{array}{c}\mathbf{S} 3000 / \mathbf{S} 3500 \\
\text { S3754 }\end{array}$ \\
\hline
\end{tabular}

\begin{tabular}{|c|c|}
\hline Data Item & Value \\
\hline MV(um): & 15.41 \\
\hline MN(um): & 0.442 \\
\hline MA(um): & 2.486 \\
\hline CS: & 2.414 \\
\hline SD: & 13.84 \\
\hline & \\
\hline
\end{tabular}



\begin{tabular}{|c|c|}
\hline \%Tile & Size(um) \\
\hline 10.00 & 0.733 \\
\hline 20.00 & 1.772 \\
\hline 25.00 & 2.532 \\
\hline 40.00 & 6.80 \\
\hline 50.00 & 12.02 \\
\hline 60.00 & 17.01 \\
\hline 70.00 & 21.38 \\
\hline 75.00 & 23.73 \\
\hline 90.00 & 34.30 \\
\hline 95.00 & 42.88 \\
\hline
\end{tabular}

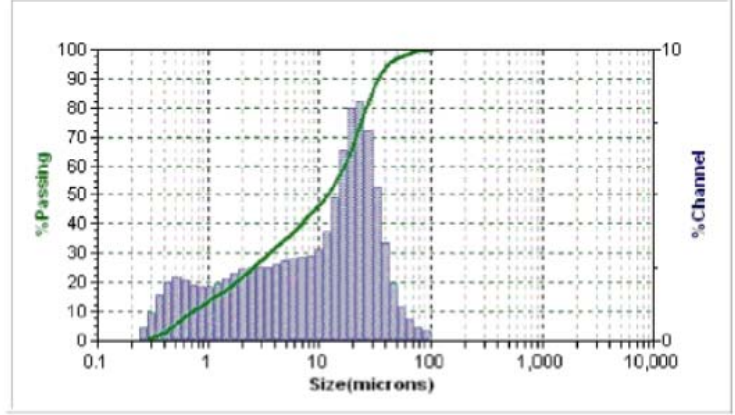

in $\mathrm{DiH2O}$

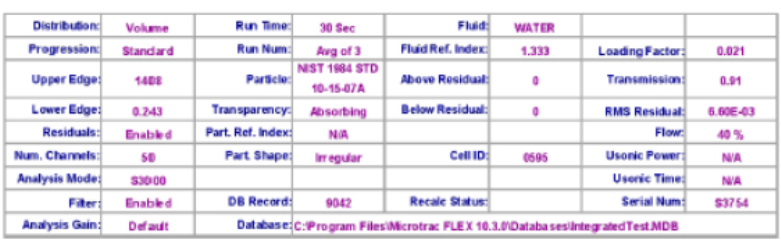

\begin{tabular}{|c|c|c|}
\hline Dia & Vol\% & Width \\
\hline 13.95 & 92.9 & 27.72 \\
\hline 0.433 & 7.1 & 0.20 \\
\hline & & \\
\hline & & \\
\hline & & \\
\hline & & \\
\hline
\end{tabular}

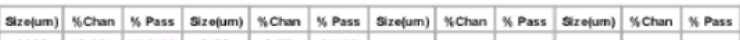
\begin{tabular}{|l|l|l|l|l|l|l|}
\hline 1408 & 0.00 & 100.00 & 3.89 & 2.53 & 31.25 \\
\hline 1184 & 0.00 & 100.00 & 3.27 & 2.52 & 28.52 \\
\hline
\end{tabular}

\begin{tabular}{|l|l|l|l|l|l|l|}
\hline 1184 & 0.00 & 100.00 & 3.27 & 2.52 & 28.72 \\
\hline
\end{tabular}

\begin{tabular}{|l|l|l|l|l|l|l|}
\hline 995.6 & 0.00 & 100.00 & 2.750 & 2.51 & 26.20 \\
\hline
\end{tabular}

\begin{tabular}{|l|l|l|l|l|l|l|}
\hline 837.2 & 0.00 & 100.00 & 2.312 & 2.44 & 23.59 \\
\hline
\end{tabular}

\begin{tabular}{|l|l|l|l|l|l|}
\hline 704.0 & 0.00 & 100.00 & 1.945 & 2.29 & 21.25 \\
\hline 5.20 & 0.00 & 100.00 & 1.65 & 2.2 & 10.96 \\
\hline
\end{tabular}

\begin{tabular}{|l|l|l|l|l|l|}
\hline 592.0 & $\mathbf{0 . 0 0}$ & 100.00 & $\mathbf{1 . 6 3 5}$ & $\mathbf{2 . 1 2}$ & 18.96 \\
\hline
\end{tabular}

\begin{tabular}{|l|l|l|l|l|l|l|}
\hline 497.8 & $\mathbf{0 . 0 0}$ & 100.00 & $\mathbf{1 . 3 7 5}$ & 1.95 & 16.84 \\
\hline
\end{tabular}

\begin{tabular}{|l|l|l|l|l|l|l|l|}
\hline $\mathbf{4 1 8 . 6}$ & $\mathbf{0 . 0 0}$ & 100.00 & $\mathbf{1 . 1 5 6}$ & $\mathbf{1 . 8 5}$ & 14.89 \\
\hline 352.0 & 0.00 & 100.00 & 0.992 & 1.34 & 13.04 \\
\hline
\end{tabular}

\begin{tabular}{|l|l|l|l|l|l|l|}
\hline 352.0 & 0.00 & 100.00 & 0.972 & 1.84 & 13.04 \\
\hline 2060 & 0.00 & 100.00 & 0.818 & 1.92 & 1120 \\
\hline
\end{tabular}

\begin{tabular}{|l|l|l|l|l|l|l|}
\hline 296.0 & 0.00 & 100.00 & 0.818 & 1.92 & 11.20 \\
\hline
\end{tabular}

\begin{tabular}{|l|l|l|l|l|l|}
\hline 248.9 & 0.00 & 100.00 & 0.688 & 2.08 & 9.28 \\
\hline & 0.00 & 100.00 & 0.578 & 2.18 & 7.20 \\
\hline
\end{tabular}

\begin{tabular}{|l|l|l|l|l|l|}
\hline $\mathbf{2 0 9 . 3}$ & $\mathbf{0 . 0 0}$ & 100.00 & $\mathbf{0 . 5 7 8}$ & $\mathbf{2 . 1 8}$ & $\mathbf{7 . 2 0}$ \\
\hline & 0.00 & 1000 & 0.456 & 2.09 & 5.02 \\
\hline
\end{tabular}

\begin{tabular}{|l|l|l|l|l|l|l|}
\hline 176.0 & 0.00 & 100.00 & 0.486 & 2.03 & 5.02 \\
\hline
\end{tabular}

\begin{tabular}{|l|l|l|l|l|l|l|}
\hline 148.0 & 0.00 & 100.00 & 0.409 & 1.56 & 2.99 \\
\hline
\end{tabular}

\begin{tabular}{|l|l|l|l|l|l|l|}
\hline 124.5 & 0.00 & 100.00 & 0.344 & 0.96 & 1.43 \\
\hline
\end{tabular}

\begin{tabular}{|l|l|l|l|l|l|l|}
\hline 104.7 & 0.31 & 100.00 & 0.2890 & 0.47 & 0.47 \\
\hline
\end{tabular}

\begin{tabular}{|c|c|c|c|}
\hline 88.00 & 0.45 & 99.69 \\
\hline
\end{tabular}

\begin{tabular}{|l|l|l|l|}
\hline 74.00 & 0.70 & 99.24 \\
\hline
\end{tabular}

\begin{tabular}{|l|l|l|l|}
\hline 62.23 & 1.15 & 98.54 \\
\hline
\end{tabular}

\begin{tabular}{|l|l|l|}
\hline 52.33 & 1.98 & 97.39 \\
\hline
\end{tabular}

\begin{tabular}{|l|l|l|}
\hline 44.00 & 3.35 & 95.41 \\
\hline
\end{tabular}

\begin{tabular}{|l|l|l|l|}
\hline 37.00 & 5.25 & 92.06 \\
\hline
\end{tabular}

\begin{tabular}{|l|l|l|}
\hline 31.11 & 7.23 & 86.81 \\
\hline
\end{tabular}

\begin{tabular}{|l|l|l|l|}
\hline 26.16 & 8.19 & 79.58 \\
\hline
\end{tabular}

\begin{tabular}{|l|l|l|l|}
\hline 22.00 & 8.01 & 71.39 \\
\hline
\end{tabular}

\begin{tabular}{|l|l|l|}
\hline 18.50 & 6.54 & 63.38 \\
\hline
\end{tabular}

\begin{tabular}{|l|l|l|}
\hline 15.56 & 4.90 & 56.84 \\
\hline
\end{tabular}

\begin{tabular}{|l|l|l|l|}
\hline 13.08 & 3.73 & 51.94 \\
\hline
\end{tabular}

\begin{tabular}{|l|l|l|}
\hline 11.00 & 3.12 & 48.21 \\
\hline
\end{tabular}

\begin{tabular}{|l|l|l|}
\hline 9.25 & 2.89 & 45.09 \\
\hline 7.70 & 2.84 & 42.20 \\
\hline
\end{tabular}

\begin{tabular}{|l|l|l|}
\hline 7.78 & 2.84 & 42.20 \\
\hline
\end{tabular}

\begin{tabular}{|l|l|l|l|}
\hline 6.54 & 2.80 & 39.36 \\
\hline 5.50 & 2.71 & 3.56
\end{tabular}

\begin{tabular}{|l|l|l|l|}
\hline 5.50 & 2.71 & 36.56 \\
\hline
\end{tabular}

\begin{tabular}{|l|l|l|}
\hline 4.62 & 2.60 & 33.85 \\
\hline
\end{tabular}

Warnings: NONE 
SRNL-STI-2012-00239

Revision 0

\section{PSD for Yellow Sand in $\mathrm{H} 2 \mathrm{O}$ and with no sonication}

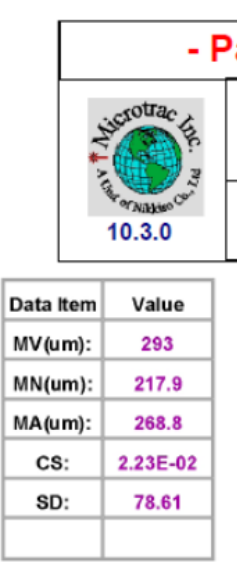

\section{Particle Size Analysis 298136 Williams \\ Safety Sand As Received}

$2012 / 03 / 29 \quad 09: 43$

S3000/S3500 S3754

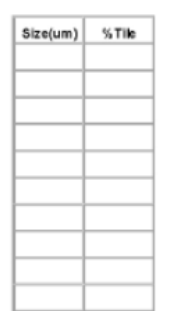

\begin{tabular}{|c|c|}
\hline \%Tile & Size(um) \\
\hline 10.00 & 195.1 \\
\hline 20.00 & 224.1 \\
\hline 25.00 & 235.5 \\
\hline 40.00 & 265.3 \\
\hline 50.00 & 284.2 \\
\hline 60.00 & 304.5 \\
\hline 70.00 & 327.5 \\
\hline 75.00 & 340.7 \\
\hline 90.00 & 401.2 \\
\hline 95.00 & 444.6 \\
\hline
\end{tabular}

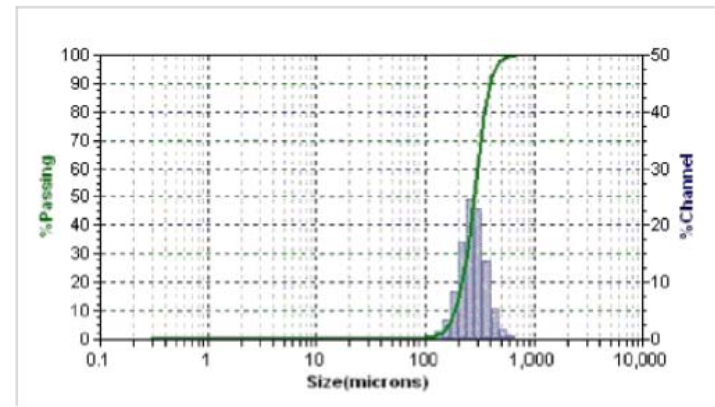

in $\mathrm{DiH} 2 \mathrm{O}$

\begin{tabular}{|c|c|c|c|c|c|c|c|}
\hline Distributong & Volume & Rin Mene: & $30 \mathrm{Sec}$ & Fluat: & Water & & \\
\hline Progrestion & Standard & Rin Num: & Arg of 3 & Flidrect index & 1.33 & Loading Factor: & 0.284 \\
\hline Upper Edge: & 1008 & Parracte: & $\begin{array}{c}\text { NST } 1984 \text { STD } \\
\text { 10.15.07A }\end{array}$ & Abow Restiduat: & 0 & Tranumassion: & 0.97 \\
\hline Lower Edoge: & 0.243 & Transparemcy: & Nosoltiby & Below Residuat: & 0 & RMS Residuat: & 7.11Ees \\
\hline Residulats: & Enabud & Part Ret. Index: & Na & & & Flow: & 405 \\
\hline Mum, Charmets: & so & Part shape: & Ireguar & Centio: & 0695 & Usonic Power: & NA \\
\hline Analyyis Mode: & 38000 & & & & & Useric Time: & NA \\
\hline Fater: & Enabikd & DB Record: & 9074 & Recalle Statur: & & Seriax Mum: & $537 \mathrm{~s}$ \\
\hline Andyris Gin: & Defact & Datthuse: & ogram File: & TE FEX & Oxatabs & GededreatMDE & \\
\hline
\end{tabular}

\begin{tabular}{|c|c|c|}
\hline Dia & Vol\% & Width \\
\hline 284.2 & 100.0 & 157.20 \\
\hline & & \\
\hline & & \\
\hline & & \\
\hline & & \\
\hline & & \\
\hline
\end{tabular}

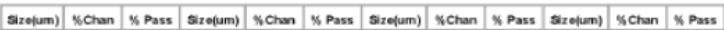
\begin{tabular}{|l|l|l|l|l|l|}
\hline $\mathbf{1 4 0 8}$ & $\mathbf{0 . 0 0}$ & 100.00 & $\mathbf{3 . 8 9}$ & $\mathbf{0 . 0 0}$ & $\mathbf{0 . 0 0}$ \\
\hline
\end{tabular}

\begin{tabular}{|l|l|l|l|l|l|}
\hline 1184 & 0.00 & 100.00 & 3.27 & 0.00 & 0.00 \\
\hline 995.6 & 0.00 & 10900 & 2.750 & 0.00 & 0.00 \\
\hline
\end{tabular}

\begin{tabular}{|l|l|l|l|l|l|l|}
\hline 995.6 & 0.00 & 100.00 & 2.750 & 0.00 & 0.00 \\
\hline
\end{tabular}

\begin{tabular}{|l|l|l|l|l|l|l|}
\hline 837.2 & 0.00 & 100.00 & 2.312 & 0.00 & 0.00 \\
\hline
\end{tabular}

\begin{tabular}{|l|l|l|l|l|l|l|}
\hline 704.0 & 0.47 & 100.00 & 1.945 & 0.00 & 0.00 \\
\hline 592.0 & 1.60 & 59.53 & 1.055 & 0.00 & 0.00 \\
\hline
\end{tabular}

\begin{tabular}{|l|l|l|l|l|l|l|}
\hline 592.0 & 1.66 & 99.53 & 1.635 & 0.00 & 0.00 \\
\hline
\end{tabular}

\begin{tabular}{|l|l|l|l|l|l|l|}
\hline 497.8 & 5.38 & 97.87 & 1.375 & 0.00 & 0.00 \\
\hline
\end{tabular}

\begin{tabular}{|l|l|l|l|l|l|l|}
\hline 418.6 & 13.65 & 92.49 & 1.156 & 0.00 & 0.00 \\
\hline
\end{tabular}

\begin{tabular}{|l|l|l|l|l|l|l|}
\hline 352.0 & 22.90 & 78.84 & 0.972 & 0.00 & 0.00 \\
\hline
\end{tabular}

\begin{tabular}{|l|l|l|l|l|l|l|}
\hline 296.0 & 24.54 & 55.94 & 0.818 & 0.00 & 0.00 \\
\hline & 17.16 & 31.00 & 0.658 & 0.00 & 0.00 \\
\hline
\end{tabular}

\begin{tabular}{|l|l|l|l|l|l|l|}
\hline 248.9 & 17.16 & 31.40 & 0.688 & 0.00 & 0.00 \\
\hline
\end{tabular}

\begin{tabular}{|l|l|l|l|l|l|l|}
\hline 209.3 & 8.44 & 14.24 & 0.578 & 0.00 & 0.00 \\
\hline 17.0 & 3.42 & 5.80 & 0.486 & 0.00 & 0.00 \\
\hline
\end{tabular}

\begin{tabular}{|l|l|l|l|l|l|}
\hline 176.0 & 3.42 & 5.80 & 0.486 & 0.00 & 0.00 \\
\hline 16.0 & 1.30 & 2.5 & 0.09 & 0.00 & 0.00 \\
\hline
\end{tabular}

\begin{tabular}{|l|l|l|l|l|l|}
\hline 148.0 & 1.38 & 2.38 & 0.409 & 0.00 & 0.00 \\
\hline
\end{tabular}

\begin{tabular}{|l|l|l|l|l|l|}
\hline 124.5 & 0.64 & 1.00 & 0.344 & 0.00 & 0.00 \\
\hline
\end{tabular}

\begin{tabular}{|l|l|l|l|l|l|}
\hline 104.7 & 0.36 & 0.36 & 0.2890 & 0.00 & 0.00 \\
\hline
\end{tabular}

\begin{tabular}{|l|l|l|}
\hline 88.00 & 0.00 & 0.00 \\
\hline 74.00 & 0.00 & 0.00 \\
\hline
\end{tabular}

\begin{tabular}{|l|l|l|}
\hline 74.00 & 0.00 & 0.00 \\
\hline
\end{tabular}

\begin{tabular}{|l|l|l|}
\hline 62.23 & 0.00 & 0.00 \\
\hline 5233 & 0.00 & 0.00 \\
\hline
\end{tabular}

\begin{tabular}{|l|l|l|l|}
\hline 52.33 & 0.00 & 0.00 \\
\hline
\end{tabular}

\begin{tabular}{|l|l|l|l|}
\hline 44.00 & 0.00 & 0.00 \\
\hline
\end{tabular}

\begin{tabular}{|l|l|l|}
\hline 37.00 & 0.00 & 0.00 \\
\hline 31.11 & 0.00 & 0.00
\end{tabular}

\begin{tabular}{|l|l|l|l|}
\hline 31.11 & 0.00 & 0.00 \\
\hline 26.16 & 0.00 & 0.00
\end{tabular}

\begin{tabular}{|l|l|l|}
\hline 26.16 & 0.00 & 0.00 \\
\hline
\end{tabular}

\begin{tabular}{|l|l|l|}
\hline 22.00 & 0.00 & 0.00 \\
\hline 10.50 & 0.00 & 0.00
\end{tabular}

\begin{tabular}{|l|l|l|}
\hline 18.50 & 0.00 & 0.00 \\
\hline
\end{tabular}

\begin{tabular}{|l|l|l|}
\hline 15.56 & 0.00 & 0.00 \\
\hline 15 & 0.00 & 0.00 \\
\hline
\end{tabular}

\begin{tabular}{|l|l|l|}
\hline 13.08 & 0.00 & 0.00 \\
\hline 1100 & 0.00 & 0.00 \\
\hline
\end{tabular}

\begin{tabular}{|l|l|l|}
\hline 11.00 & 0.00 & 0.00 \\
\hline
\end{tabular}

\begin{tabular}{|l|l|l|}
\hline $\mathbf{9 . 2 5}$ & $\mathbf{0 . 0 0}$ & 0.00 \\
\hline 7.00 & 0.00 & 0.00 \\
\hline
\end{tabular}

\begin{tabular}{|l|l|l|}
\hline 7.78 & 0.00 & 0.00 \\
\hline
\end{tabular}

\begin{tabular}{|l|l|l|}
\hline 6.54 & 0.00 & 0.00 \\
\hline 5.50 & 0.00 & 0.00 \\
\hline
\end{tabular}

\begin{tabular}{|l|l|l|l|}
\hline 5.50 & 0.00 & 0.00 \\
\hline
\end{tabular}

\begin{tabular}{|l|l|l|}
\hline 5.50 & 0.00 & 0.00 \\
\hline 4.62 & 0.00 & 0.00 \\
\hline
\end{tabular}

Warnings: NONE 


\section{PSD for Stainless Steel in 50\% Isopropyl no sonication}
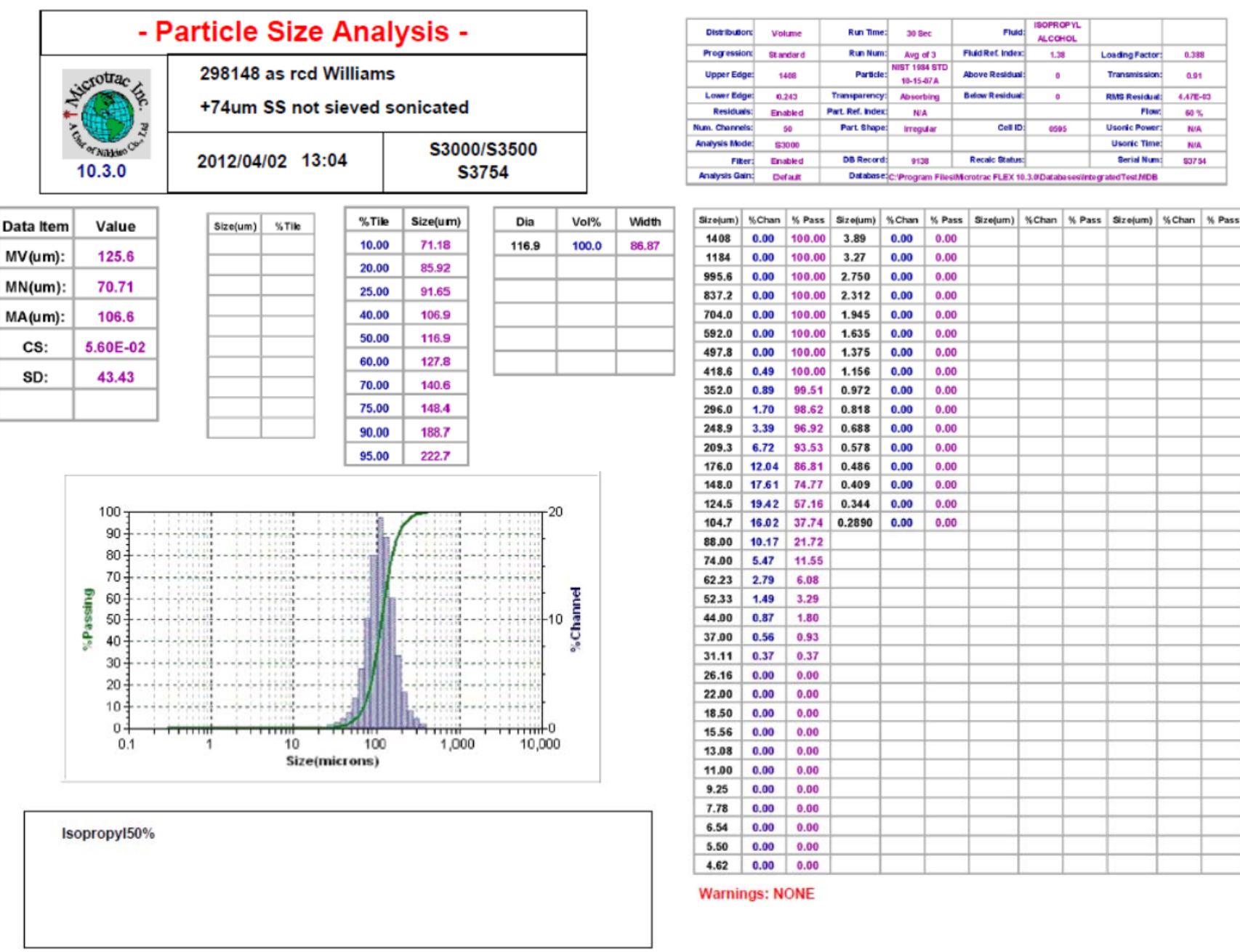

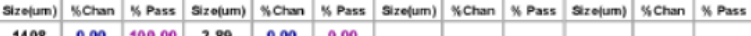

\begin{tabular}{|l|l|l|l|l|l|l|}
\hline 1184 & 0.00 & 100.00 & 3.27 & 0.00 & 0.00 \\
\hline
\end{tabular}

\begin{tabular}{|l|l|l|l|l|l|}
\hline $\mathbf{9 9 5 . 6}$ & $\mathbf{0 . 0 0}$ & 100.00 & 2.750 & 0.00 & 0.00 \\
\hline
\end{tabular}

\begin{tabular}{|l|l|l|l|l|l|}
\hline $\mathbf{9 9 5 . 6}$ & $\mathbf{0 . 0 0}$ & 100.00 & 2.750 & 0.00 & 0.00 \\
\hline $\mathbf{8 3 7 . 2}$ & $\mathbf{0 . 0 0}$ & 100.00 & $\mathbf{2 . 3 1 2}$ & $\mathbf{0 . 0 0}$ & 0.00 \\
\hline & 0.00 & 100.00 & 1.945 & 0.00 & 0.00 \\
\hline
\end{tabular}

\begin{tabular}{|l|l|l|l|l|l|}
\hline 704.0 & 0.00 & 100.00 & 1.945 & 0.00 & 0.00 \\
\hline
\end{tabular}

\begin{tabular}{|l|l|l|l|l|l|l|}
\hline 592.0 & 0.00 & 100.00 & 1.635 & 0.00 & 0.00 \\
\hline
\end{tabular}

\begin{tabular}{|l|l|l|l|l|l|l|}
\hline 497.8 & 0.00 & 100.00 & 1.375 & 0.00 & 0.00 \\
\hline
\end{tabular}

\begin{tabular}{|l|l|l|l|l|l|l|}
\hline 418.6 & 0.49 & 100.00 & 1.156 & 0.00 & 0.00 \\
\hline
\end{tabular}

\begin{tabular}{|c|c|c|c|c|c|}
\hline 352.0 & 0.89 & 99.51 & 0.972 & 0.00 & 0.00 \\
\hline 296.0 & 1.70 & 98.62 & 0.818 & 0.00 & 0.00 \\
\hline
\end{tabular}

\begin{tabular}{|l|l|l|l|l|l|}
\hline 296.0 & 1.70 & 98.62 & 0.818 & 0.00 & 0.00 \\
\hline
\end{tabular}

\begin{tabular}{|l|l|l|l|l|l|l|}
\hline 248.9 & 3.39 & 96.92 & 0.688 & 0.00 & 0.00 \\
\hline
\end{tabular}

\begin{tabular}{|l|l|l|l|l|l|l|}
\hline 209.3 & 6.72 & 93.53 & 0.578 & 0.00 & 0.00 \\
\hline
\end{tabular}

\begin{tabular}{|l|l|l|l|l|l|}
\hline 176.0 & 12.04 & 86.81 & 0.486 & 0.00 & 0.00 \\
\hline
\end{tabular}

\begin{tabular}{|l|l|l|l|l|l|}
\hline 148.0 & 17.61 & 74.77 & 0.409 & 0.00 & 0.00 \\
\hline
\end{tabular}

\begin{tabular}{|l|l|l|l|l|l|l|}
\hline 124.5 & 19.42 & 57.16 & 0.344 & 0.00 & 0.00 \\
\hline 10.7 & 16.02 & 37.74 & 0.2090 & 0.00 & 0.00 \\
\hline
\end{tabular}

\begin{tabular}{|l|l|l|l|l|l|l|}
\hline 104.7 & 16.02 & 37.74 & 0.2890 & 0.00 & 0.00 \\
\hline
\end{tabular}

\begin{tabular}{|l|l|l|l|}
\hline 88.00 & 10.17 & 21.72 \\
\hline 74.00 & 5.47 & 1.55
\end{tabular}

\begin{tabular}{|l|l|l|}
\hline 74.00 & 5.47 & 11.55 \\
\hline
\end{tabular}

\begin{tabular}{|l|l|l|}
\hline 62.23 & 2.79 & 6.08 \\
\hline
\end{tabular}

\begin{tabular}{|l|l|l|}
52.33 & 1.49 & 3.29 \\
\hline
\end{tabular}

\begin{tabular}{|l|l|l|}
\hline 44.00 & 0.87 & 1.80 \\
\hline
\end{tabular}

\begin{tabular}{|l|l|l|}
\hline 37.00 & 0.56 & 0.93 \\
\hline
\end{tabular}

\begin{tabular}{|l|l|l|l|}
\hline 31.11 & 0.37 & 0.37 \\
\hline
\end{tabular}

\begin{tabular}{|l|l|l|}
\hline 26.16 & 0.00 & 0.00 \\
\hline
\end{tabular}

\begin{tabular}{|l|l|l|}
\hline 22.00 & 0.00 & 0.00 \\
\hline
\end{tabular}

\begin{tabular}{|l|l|l|}
\hline $\mathbf{1 8 . 5 0}$ & $\mathbf{0 . 0 0}$ & 0.00 \\
\hline
\end{tabular}

\begin{tabular}{|l|l|l|}
\hline 15.56 & 0.00 & 0.00 \\
\hline 15.00
\end{tabular}

\begin{tabular}{|l|l|l|l|}
\hline 13.08 & 0.00 & 0.00 \\
\hline 1100 & 0.00 & 0.00 \\
\hline
\end{tabular}

\begin{tabular}{|l|l|l|}
\hline 11.00 & 0.00 & 0.00 \\
\hline
\end{tabular}

\begin{tabular}{|l|l|l|}
\hline 9.25 & 0.00 & 0.00 \\
\hline
\end{tabular}

\begin{tabular}{|l|l|l|}
\hline 7.78 & 0.00 & 0.00 \\
\hline
\end{tabular}

\begin{tabular}{|l|l|l|}
\hline 6.54 & 0.00 & 0.00 \\
\hline 5.50 & 0.00 & 0.00 \\
\hline
\end{tabular}

\begin{tabular}{|l|l|l|}
\hline 5.50 & 0.00 & 0.00 \\
\hline
\end{tabular}

\begin{tabular}{|l|l|l|}
\hline 4.62 & 0.00 & 0.00 \\
\hline
\end{tabular}

Warnings: NONE 
SRNL-STI-2012-00239

Revision 0

PSD for Stainless Steel in 50\% Isopropyl and with sonication

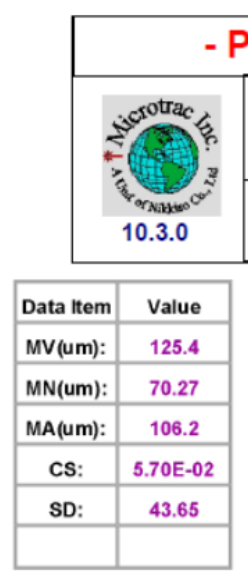

- Particle Size Analysis -

298149 as red Williams

+74um SS not sieved not sonic

\begin{tabular}{|l|l|l}
$2012 / 04 / 02$ & $12: 32$ & S3000/S3500
\end{tabular}

S3754

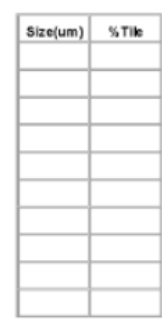

\begin{tabular}{|c|c|}
\hline \%Tile & Size(um) \\
\hline 10.00 & 70.69 \\
\hline 20.00 & 85.45 \\
\hline 25.00 & 91.19 \\
\hline 40.00 & 106.5 \\
\hline 50.00 & 116.5 \\
\hline 60.00 & 127.4 \\
\hline 70.00 & 140.3 \\
\hline 75.00 & 148.2 \\
\hline 90.00 & 188.9 \\
\hline 95.00 & 223.4 \\
\hline
\end{tabular}

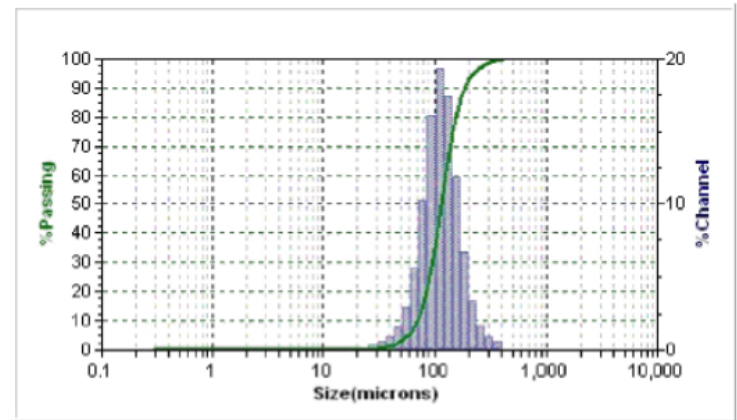

Isopropyl50\%

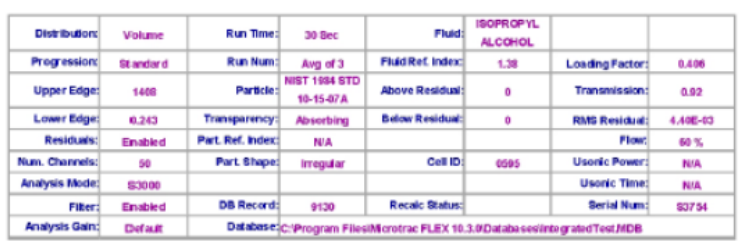

\begin{tabular}{|c|c|c|}
\hline Dia & Vol\% & Width \\
\hline 116.5 & 100.0 & 87.30 \\
\hline & & \\
\hline & & \\
\hline & & \\
\hline & & \\
\hline & & \\
\hline
\end{tabular}


\begin{tabular}{|l|l|l|l|l|l|}
\hline 1408 & 0.00 & 100.00 & 3.89 & 0.00 & 0.00 \\
\hline & 0.00 & 10.00 & 3.27 & 0.00 & 0.00 \\
\hline
\end{tabular} \begin{tabular}{|l|l|l|l|l|l|l|}
\hline 1184 & 0.00 & 100.00 & 3.27 & 0.00 & 0.00 \\
\hline 95.6 & 0.00 & 100.00 & 2.750 & 0.00 & 0.00 \\
\hline
\end{tabular}

\begin{tabular}{|l|l|l|l|l|l|l|}
\hline 995.6 & 0.00 & 100.00 & 2.750 & 0.00 & 0.00 \\
\hline 5972 & 0.00 & 10.00 & 2.312 & 0.00 & 0.00 \\
\hline
\end{tabular}

\begin{tabular}{|l|l|l|l|l|l|}
\hline 837.2 & 0.00 & 100.00 & 2.312 & 0.00 & 0.00 \\
\hline
\end{tabular}

\begin{tabular}{|l|l|l|l|l|l|l|}
\hline 704.0 & 0.00 & 100.00 & 1.945 & 0.00 & 0.00 \\
\hline
\end{tabular}

\begin{tabular}{|l|l|l|l|l|l|l|}
\hline 592.0 & 0.00 & 100.00 & 1.635 & 0.00 & 0.00 \\
\hline
\end{tabular}

\begin{tabular}{|l|l|l|l|l|l|}
\hline $\mathbf{4 9 7 . 3}$ & $\mathbf{0 . 0 0}$ & 100.00 & $\mathbf{1 . 3 7 5}$ & $\mathbf{0 . 0 0}$ & 0.00 \\
\hline
\end{tabular}

\begin{tabular}{|l|l|l|l|l|l|}
\hline 418.6 & 0.51 & 100.00 & 1.156 & 0.00 & 0.00 \\
\hline & 0.91 & 0.95 & 0.972 & 0.00 & 0.00 \\
\hline
\end{tabular}

\begin{tabular}{|l|l|l|l|l|l|l|}
\hline 352.0 & 0.91 & 99.49 & 0.972 & 0.00 & 0.00 \\
\hline
\end{tabular}

\begin{tabular}{|l|l|l|l|l|l|l|}
\hline 296.0 & 1.72 & 98.58 & 0.818 & 0.00 & 0.00 \\
\hline
\end{tabular}

\begin{tabular}{|l|l|l|l|l|l|}
\hline 248.9 & 3.40 & 96.86 & 0.688 & 0.00 & 0.00 \\
\hline
\end{tabular}

\begin{tabular}{|l|l|l|l|l|l|}
\hline 209.3 & 6.66 & 93.46 & 0.578 & 0.00 & 0.00 \\
\hline
\end{tabular}

\begin{tabular}{|l|l|l|l|l|l|l|}
\hline 176.0 & 11.89 & 86.80 & 0.486 & 0.00 & 0.00 \\
\hline
\end{tabular}

\begin{tabular}{|l|l|l|l|l|l|l|}
\hline 148.0 & $17 A 2$ & 74.91 & 0.409 & 0.00 & 0.00 \\
\hline
\end{tabular}

\begin{tabular}{|l|l|l|l|l|l|l|}
\hline 124.5 & 19.32 & 57.49 & 0.344 & 0.00 & 0.00 \\
\hline
\end{tabular}

\begin{tabular}{|l|l|l|l|l|l|l|}
\hline 104.7 & 16.05 & 38.17 & 0.2890 & 0.00 & 0.00 \\
\hline 0.0 & 10.20 & 2.12 & & & \\
\hline
\end{tabular}

\begin{tabular}{|l|l|l|l|}
\hline 88.00 & 10.28 & 22.12 \\
\hline
\end{tabular}

\begin{tabular}{|l|l|l|}
\hline 74.00 & 5.58 & 11.84 \\
\hline
\end{tabular}

\begin{tabular}{|l|l|l|}
\hline 62.23 & 2.87 & 6.26 \\
\hline 52.33 & 1.54 & 3.39 \\
\hline
\end{tabular}

\begin{tabular}{|l|l|l|}
\hline 52.33 & 1.54 & 3.39 \\
\hline & 0.92 & 1.05
\end{tabular}

\begin{tabular}{|l|l|l|}
\hline $\mathbf{4 4 . 0 0}$ & $\mathbf{0 . 9 0}$ & $\mathbf{1 . 8 5}$ \\
\hline
\end{tabular}

\begin{tabular}{|l|l|l|l|}
\hline $\mathbf{3 7 . 0 0}$ & $\mathbf{0 . 5 7}$ & 0.95 \\
\hline
\end{tabular}

\begin{tabular}{|l|l|l|}
\hline 31.11 & 0.38 & 0.38 \\
\hline
\end{tabular}

\begin{tabular}{|l|l|l|}
\hline 26.16 & 0.00 & 0.00 \\
\hline
\end{tabular}

\begin{tabular}{|l|l|l|}
\hline 22.00 & 0.00 & 0.00 \\
\hline
\end{tabular}

\begin{tabular}{|l|l|l|}
\hline 18.50 & 0.00 & 0.00 \\
\hline 15.56 & 0.00 & 0.00 \\
\hline
\end{tabular}

\begin{tabular}{|l|l|l|}
\hline 15.56 & 0.00 & 0.00 \\
\hline 130 & 0.00 & 0.00
\end{tabular}

\begin{tabular}{|l|l|l|l|}
\hline 13.08 & 0.00 & 0.00 \\
\hline 11.00 & 0.00 & 0.00 \\
\hline
\end{tabular}

\begin{tabular}{|l|l|l|}
\hline 11.00 & $\mathbf{0 . 0 0}$ & $\mathbf{0 . 0 0}$ \\
\hline
\end{tabular}

\begin{tabular}{|l|l|l|}
\hline 9.25 & 0.00 & 0.00 \\
\hline 7.78 & 0.00 & 0.00 \\
\hline
\end{tabular}

\begin{tabular}{|l|l|l|}
\hline 7.78 & 0.00 & 0.00 \\
\hline 6.54 & 0.09 & 0.00
\end{tabular}

\begin{tabular}{|l|l|l|}
\hline 6.54 & 0.00 & 0.00 \\
\hline 5.50 & 0.00 & 0.00 \\
\hline
\end{tabular}

\begin{tabular}{|l|l|l|}
\hline 5.50 & 0.00 & 0.00 \\
\hline 5.50 & 0.00 & 0.00 \\
\hline
\end{tabular}

\begin{tabular}{|l|l|l|}
\hline 5.50 & 0.00 & 0.00 \\
\hline 4.62 & 0.00 & 0.00 \\
\hline
\end{tabular}

Warnings: NONE 


\section{Distribution:}
A. B. Barnes, 999-W
B. J. Giddings, 786-5A
C. C. Herman, $999-\mathrm{W}$
S. L. Marra, 773-A
F. M. Pennebaker, 773-42A
W. R. Wilmarth, 773-A
M. R. Duignan, 786-5A
T. J. Steeper, 786-5A
J. S. Steimke, 786-5A
M. D. Fowley, 786-5A
D. J. Adamson, 999-W
M. G. Thien, Washington River Protection Solutions-Hanford
T. A. Wooley, Washington River Protection Solutions-Hanford
K. P. Lee, Washington River Protection Solutions-Hanford 